\title{
Conservation of Translation Initiation Between Prokaryotes and Eukaryotes
}

\author{
by
}

Nathalie Puchacz

A thesis submitted to the Faculty of Graduate and Postdoctoral Affairs in partial fulfillment of the requirements for the degree of

Master of Science

in

Biology

Carleton University

Ottawa, Ontario

(C) 2019

Nathalie Puchacz 


\begin{abstract}
While the fundamental goals of translation initiation are the same for all cells, it is the most phylogenetically diverse step within translation. Until recently, there hasbeen no evidence of a molecular mechanism that can initiate translation in both prokaryotes and eukaryotes. Colussi et al. (2015) reported that the eukaryotic PSIV IGR IRES can initiate prokaryotic translation and effectively circumvent domain-specific divergences. This novel discovery led us to investigate whether this IRES is unique in its ability to initiate prokaryotic translation and attempt to elucidate any distinguishing characteristics that allow for its mechanism of action to occur. Our results indicate that this IRES is unique in its ability to initiate prokaryotic translation. It also appears that the structural integrity of this IRES is less critical in prokaryotes than eukaryotes, and complementary interactions between Domain III and the 16SrRNA may contribute to this IRES' mechanism of action.
\end{abstract}




\section{Acknowledgments}

So many people deserve recognition for their contribution to this thesis. Aboveall, to Dr. Ashkan Golshani, thank you. Not only for the opportunity to conduct this research, but for also accepting me into your lab, for your constant support (both in the lab and life) and for furthering me as a researcher.

To my fellow Golshani lab members, past and present, thank you for your support, your advice and your acceptance. Thank you for becoming my family.

To my friends and family, thank you for your support, patience and understanding when life got tough.

And finally, to James. Words can't even begin to describe how grateful I am for your presence in my life. Thank you for your support through failed experiments, the struggles of writing, the pressures of life and the-day-to-day mood swings. I don't think I could have done this without you. 


\section{Table of Contents}

\begin{tabular}{lr}
\hline Abstract & ii \\
\hline Acknowle dgme nts & iii \\
\hline Table of Contents & iv \\
\hline List of Illus trations & vi \\
\hline List of Abbre viations & vii \\
\hline & \\
1. Gene ral Introduction & $\mathbf{1 0}$ \\
\hline 1.1. Translation & $\mathbf{1 0}$ \\
1.1.1. Ribosomes & 10 \\
1.2. Translation Initiation & $\mathbf{1 4}$ \\
1.2.1. Eukaryotic Cap-Dependent Mechanism of Translation Initiation & 14 \\
1.2.2. Eukaryotic Cap-Independent Mechanism of Translation Initiation & 18 \\
1.2 .2 .1 . Leaky Scanning & 18 \\
1.2 .2 .2 . Ribosome Shunting & 19 \\
1.2 .2 .3 . Reinitiation & 20 \\
1.2 .2 .4 . Internal Ribosome Entry Site & 21 \\
1.2.3. Prokaryotic Mechanism of Translation Initiation & 28 \\
1.2.1. Shine-Dalgarno and Enhanc er Sequences & 30 \\
1.3. Proposed Similarities betwe en Prok aryotic \& Euk aryotic Translation Initiation & $\mathbf{3 1}$ \\
1.4. Focus and Objectives & $\mathbf{3 2}$
\end{tabular}

2. Journal Article 36

2.1. Introduction

2.2.1. Organization of Dic istronic Reporter Plasmid 40

2.2.2. Cloning of Dual-Luciferase Constructs 41

2.2.2.1 Viral IRES, Cellular IRES, and Structured RNA Elements 41

2.2.2.2. Mutated Domain III Elements 41

2.2.3. Bacterial Cell Cultures $\quad 42$

2.2.4. Luciferase Induction and Measurements $\quad 42$

2.2.5. Statistical Analyses $\quad 43$

2.3. Results and Discussion $\quad 45$

2.3.1. Capabilities of Eukaryotic IRESs to Initiate Prokaryotic Translation 46

2.3.2. Truncation of Domain III 53

2.3.3. Mutating Complementary Regions $\quad 56$

$\begin{array}{ll}\text { 2.4. Conclusion } & 61\end{array}$

\begin{tabular}{lr} 
3. General Discussion & 62 \\
\hline .1. Capabilitios
\end{tabular}

3.1. Capabilities of Eukaryotic IRESs to Initiate Prokaryotic Translation $\quad 62$

3.2. Truncation of Independent Domain III 66

3.3. Induced Mutations of Complementary Regions $\quad 68$

$\begin{array}{lr}\text { 3.4. Conclusions } & 70\end{array}$

$\begin{array}{ll}\text { 3.5. Future Directions } & 71\end{array}$

\begin{tabular}{lr} 
4. References & 73 \\
\hline
\end{tabular}

\begin{tabular}{lr} 
5. Appendix 1 & 81 \\
\hline
\end{tabular} 


\section{List of Tables}

Table 1. Sequence of mutated Domain III inserts. .............................................. 42

Table 2. Oligonucleotide primers used in the PCR amplification of viral and cellular IRESs and structured RNA elements for their insertion into the dual-luciferase pET 30 a reporter vector. 


\section{List of Illustrations}

Figure 1. Composition of prokaryotic and eukaryotic ribosomes, and their shared conserved core..

Figure 2. Schematic diagram of cap-dependent translation initiation in eukaryotes...... 17

Figure 3. Visual depiction of viral IRES groupings and their requirements to initiate translation, compared with canonical cap-dependent translation initiation..................22

Figure 4. Schematic diagram of prokaryotic translation initiation......................... 29

Figure 5. The dual-luciferase reporter construct within the modified pET 30 a vector utilized.

Figure 6. RNAfold predicted MFE secondary structures of the yeast cellular IRES elements used. 47

Figure 7. Secondary structures of viral IRES elements used. 48

Figure 8. RNAfold predicted MFE secondary structures of reverse inserted yeast cellular IRES elements used. 50

Figure 9. Normalized fold change of luciferase expression illustrating the difference in ability of various IRES and RNA elements to express FLuc relative to the SD and enhancer controlled RLuc, compared to the PSIV construct, within E. coli. 52

Figure 10. (A) Visual representation of the PSIV Full-Length Domain III. Sequences forming Domains I and II were deleted. (B) Visual representation of the PSIVT runcated Domain III, whereby the first 11 nucleotides were removed from the 5' end of the FullLength Domain III sequence.

Figure 11. Normalized fold change of luciferase expression illustrating the difference in ability of the Full-Length and Truncated Domain III elements to express FLuc relative to the SD and enhancer controlled RLuc, compared to the PSI V construct, within E. coli. 55

Figure 12. Complementarity illustrated between nucleotides 932-938 and 1465-1471 of the 16S rRNA of E. coli and Domain III of the PSIV IGR IRES.

Figure 13. (A) Visual representation of the Truncated Domain III bearing the introduced mutations CACT within Box I. (B) Visual representation of the Truncated Domain III bearing the introduced mutations ACTT within Box 2 . 59

Figure 14. Normalized fold change of luciferase expression illustrating the difference in ability of mutated T runcated Domain III elements, CACT and ACT T, compared to the Truncated Domain III, to express FLuc relative to the SD and enhancer controlled RLuc, within E. coli. 60 


\section{List of Abbreviations}

A - adenine

ANOVA - analysis of variance

APAF-1 - apoptotic protease activating factor 1

A-Site - aminoacyl/acceptor site

BCL-2 - B-cell lymphoma 2

BVDV - Bovine viral diarrhea virus

$\mathrm{C}-$ cytosine

CaMV - Cauliflower mosaic virus

c-myc-c-myelocytoma

CrPV - Cricket paralysis virus

CSFV - Classical swine fever virus

DNA - deoxyribonucleic acid

eIFs - eukaryotic initiation factors

EMCV - Encephalomyocarditis virus

eRF1 - eukaryotic release factor 1

E-Site - exit site

FLuc - Firefly luciferase

FMDV - Foot-and-Mouth disease virus

$\mathrm{G}$ - guanine

GDP - guanosine diphosphate

GT P - guanosine triphosphate

$\mathrm{HCV}$ - Hepatitis $C$ virus

HIF-1 $\alpha$ - Hypoxia-inducible factor 1 -alpha

HPV - Human papillomavirus

IAPV - Isreali acute paralysis virus

IF - initiation factor

IGF1R - insulin-like growth factor 1

IGR - intergenic

Indel - insertion/deletion

IPT G- Isopropyl-ß-D-thiogalactopyranoside

IRES - internal ribosome entry site

IT AF - IRES trans acting factor

lac $Z$ - $\beta$-galactosidase

LB - Luria Broth

Met-methionine

MFE - minimum free energy

mRNA - messenger ribonucleic acid

$\mathrm{mRNP}$ - messenger ribonucleoprotein

ORF - open reading frame 
PABP - poly-A binding protein

PBS - phosphate buffered saline

PCPB-2 - poly(C)-binding protein 2

PCR - polymerase chain reaction

PIC - pre-initiation complex

PK - pseudoknot

PLB - passive lysis buffer

PMT - photomultiplier tube

Post-TCs - post-termination complexes

P-Site - peptidyl site

PSIV - Plautia stali intestinal virus

PTB - polypyrimidine-tract binding protein

PTC - peptidyl transferase center

PV - Poliovirus

RBS - ribosome binding site

RLU - relative light units

RLuc - Renilla luciferase

RNA - ribonucleic acid

rRNA - ribosomal ribonucleic acid

RT BV - Rice tungro bacilliform virus

$\mathrm{RV}$ - Rhinovirus

SD - Shine-Dalgarno

SL - stem-loop

sORF - short open reading frame

$\mathrm{T}$ - thymine

tRNA - transfer ribonucleic acid

$\mathrm{U}$ - uracil

uORF - upstream open reading frame

UTR - untranslated region 


\section{General Introduction}

\subsection{Translation}

The translation of messenger RNA (mRNA) into protein is the final phase within the process of gene expression. Translation is a cyclical process that can be subdividedinto four steps: initiation, elongation, termination and ribosome recycling.

Initiation marks the beginning of translation, whereby the ribosomal subunits are assembled at the initiation codon on the mRNA, with initiator transfer RNA (tRNA)bound in the ribosomal P-site and base paired with the start codon present on the mRNA (Allen \& Frank, 2007). During elongation, the mRNA codons paired with their respective tRNA are repetitively decoded and peptide bonds are formed. Termination occurs once a stop codon is encountered and the newly completed polypeptide is released from the ribosome. Within the final stage, recycling, the ribosomal subunits are dissociated, releasing the mRNA and deacylated tRNAs, which will be reused for another round of initiation (Kapp and Lorsch, 2004).

\subsubsection{Ribosomes}

In both prokaryotic and eukaryotic cells, the functional machinery operatingduring the process of translation is the ribosome. Ribosomes are dynamic ribonucleoprotein complexes that catalyze the conversion of genetic information on an mRNA template into a nascent polypeptide chain (Laursen et al., 2005). Consisting of proteins organized on a scaffolding of RNA, ribosomes are composed of a large and small subunit, each respectively possessing functional significance (Melnikov et al., 2012). The small subunit contains the conduit that mRNA is conducted along during translation, the decodingcenter, 
and the tRNA binding sites (A, P and E-sites) (Klinge et al., 2011; de la Cruz, Karbstein and Woolford, 2015). This subunit is the functional site of the decoding process, whereby tRNA is paired to its appropriate mRNA codon and promotes adequate translation fidelity by the monitoring of base pairing (Ramakrishnan, 2002). The large subunit acts as the active site of the ribosome. Significant functional sites include the peptidyl transferase center (PTC), where peptide bonds are catalyzed, the tRNA binding sites and the peptide exit tunnel, which extends throughout the subunit (de la Cruz, Karbstein and Woolford, 2015). The entirety of the ribosomal catalytic activity is carried out by the ribosomal RNA (rRNA), with ribosomal proteins largely residing on the surface of the PTC, acting to stabilize the structure (Lafontaine and Tollervey, 2001).

Ribosomes can be somewhat heterogeneous and possess distinct structural and compositional differences (Figure 1). Between species, disparities can include size, structure, protein to RNA proportions, but can also be more exclusive, including domainspecific proteins, mutations within conserved proteins, and expanded fragments of rRNAs (Melnikov et al., 2012). Regardless of the differences, a structurally conserved portion of the ribosome is present in cells from all domains (Ben-Shem et al., 2011). The core is composed of the aforementioned functional sites, such as the decoding site, PTC and tRNA-binding sites. These sites are comprised of 34 conserved proteins, nearly evenly split between the small and large subunit (15 and 19 proteins, respectively) and over four thousand RNA bases (Melnikov et al., 2012). Many of the expanded rRNA segments and protein moieties cluster on the solvent-exposed surface of the ribosome, surrounding the conserved core in an arrangement that does not directly interrupt the functional centersof ribosomes and allows them to be accessible to potential molecular interactors, such as 
chaperones and translation factors (Spahn et al., 2001; Melnikov et al., 2012; Ramesh and Woolford, 2016).

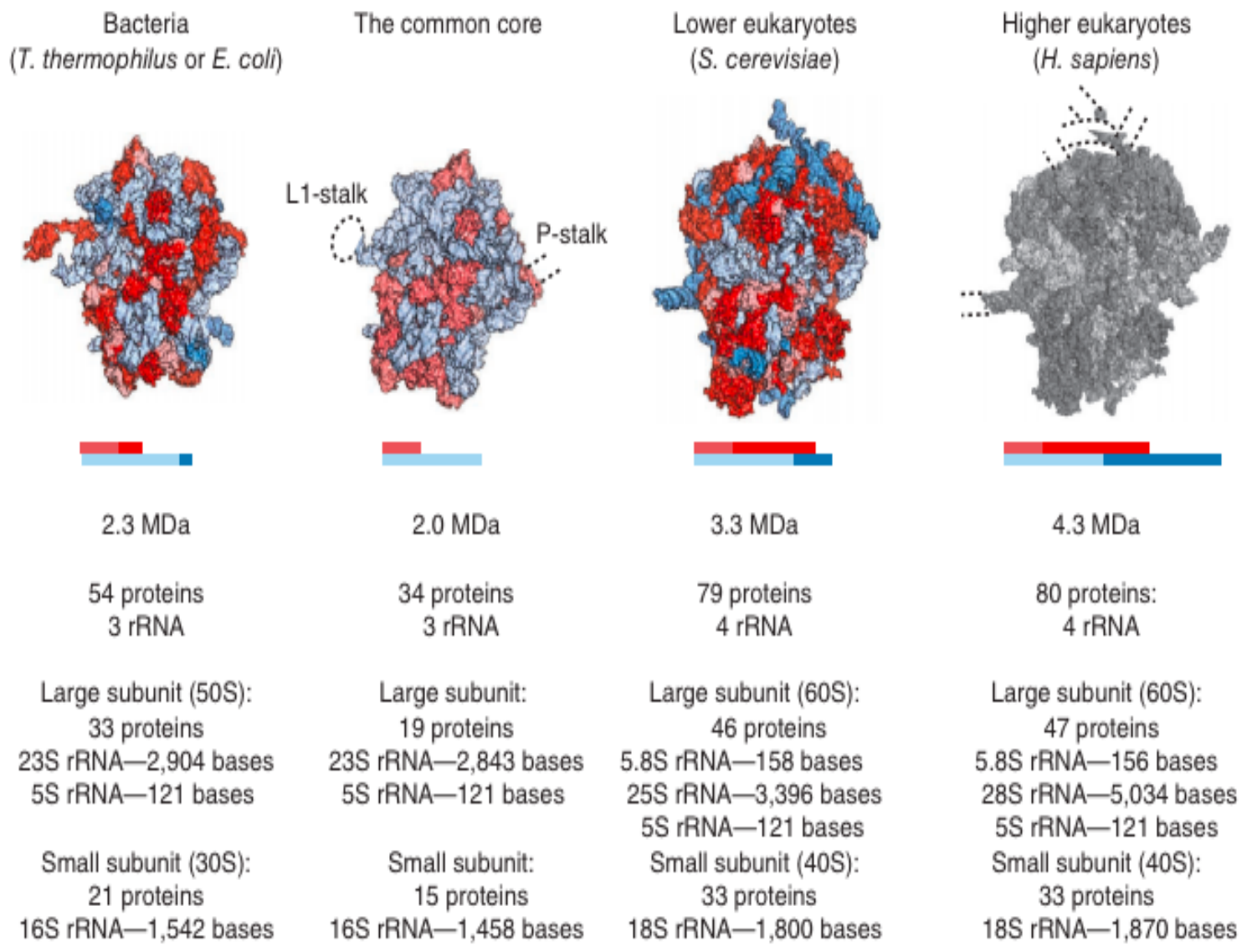

Figure 1. Composition of bacterial and eukaryotic ribosomes, and their shared conservedcore. The conserved core is composed of RNA (light blue) and proteins (light red), with additions to conserved proteins (red) and extensions of rRNA (blue). Reproduced with permission from (Melnikov et al., 2012).

In bacteria, the $70 \mathrm{~S}$ ribosome is composed of the small $30 \mathrm{~S}$ ribosomal subunit and the large 50S ribosomal subunit (Ramakrishnan, 2002). The small subunit is composed of 21 ribosomal proteins attached to the $16 \mathrm{~S}$ rRNA chain and organized into three distinct structural subdomains: the body, containing the 5' domain of the 16SrRNA; the platform, containing the central domain; and the head, which contains the 3 ' major domain (Lafontaine and T ollervey, 2001). The large subunit is composed of 33 ribosomal proteins 
accompanying two rRNA molecules: the 5S rRNA and the 23S rRNA (Ben-Shem et al., 2011). Surrounding the conserved core, the 70 S ribosome possesses 20 bacteria-specific ribosomal proteins (6 and 14 within the small and large subunits, respectively), along with their own extensions within conserved proteins and rRNA (Melnikov et al., 2012).

In eukaryotes, the $80 \mathrm{~S}$ ribosome is composed of the small $40 \mathrm{~S}$ ribosomal subunit and the large 60S ribosomal subunit (Wool, 1979). The small subunit is composed of 33 ribosomal proteins attached to the $18 \mathrm{~S}$ rRNA chain and organized into three distinct structural subdomains: the body, containing the 5' domain of the $18 \mathrm{SrRNA}$; the platform, containing the central domain; and the head, which contains the $3^{\prime}$ major domain (de la Cruz, Karbstein and Woolford, 2015). The large subunit is composed of 46 ribosomal proteins accompanying three rRNA molecules: 25S, 5.8S and 5S (Ben-Shem et al., 2011).

Compared to its bacterial counterpart, the eukaryotic ribosome is more complex. This statement is attributable to the fact that the eukaryotic ribosome accommodates an additional 46 eukaryotic-specific ribosomal proteins (18 and 28 within the small and large subunits, respectively), with homologous proteins within the conserved core having acquired supplementary insertions and extensions. Similarly, the rRNA also possesses additional blocks of sequences, known as expansion segments, interspersed throughout the core rRNA secondary structure at unambiguous, conserved sites (Jenner et al., 2012; Melnikov et al., 2012; Ramesh and Woolford, 2016). These numerous additions of expanded ribosomal elements account for the approximate 40\% larger size of the eukaryotic ribosome compared to the bacterial ribosome, as well as the increased level of complexity within the pathway of eukaryotic translation (Ben-Shem et al., 2011; Melnikov et al., 2012). 


\subsection{Translation Initiation}

Gene expression is a highly controlled process and translation initiation is a critical control point during protein synthesis. Translation initiation acts as the rate-limiting step in translation, influencing subsequent efficiency, mRNA stability and kinetics (Gray and Wickens, 1998; Jackson, Hellen and Pestova, 2010; Benelli and Londei, 2011). Though the fundamental goal of translation initiation is the same in bacteria and eukaryotes, it is the most phylogenetically diverse step within the process of translation (Laursen et al., 2005; Benelli and Londei, 2011).

\subsubsection{Eukaryotic Cap-Dependent Mechanis $m$ of Translation Initiation}

Translation initiation in eukaryotes is a highly regulated and complex process (Figure 2). Over time, eukaryotes have evolved sophisticated mechanisms, conformations and factors to initiate the process of translation. The formation of novel mRNA structures, such as the 5' methyl-guanosine cap ( $\left.{ }^{7} \mathrm{mGpppN}\right)$ and the poly-adenosine (poly-A) tail, enabled the ability of mRNA to recruit ribosomes in a multi-step process using proteinRNA and protein-protein interactions, while simultaneously maintaining controlovergene expression.

Eukaryotic canonical translation initiation is a cyclical process whereby rounds of translation initiation follow the recycling of post-termination complexes (post-TCs) present from the previous rounds of translation (Jackson, Hellen and Pestova, 2010). These complexes are composed of mRNA-bound 80 S ribosomes, P-site deacylated tRNA and a minimum of one release factor, commonly eukaryotic release factor 1 (eRF1). At low free $\mathrm{Mg}^{2+}$ concentrations, recycling is facilitated by eukaryotic initiation factors (eIFs). eIF3, 
alongside eIF1 and eIF1 A, mediate the release of the ligands and the disassociation of ribosomes into available 60S subunits, and mRNA-bound and tRNA-bound 40S subunits. Particularly, eIF1 stimulates the release of tRNA, while eIF3j mediates mRNA release, and eIF3, in concert with eIF1 and eIF1A, remain associated with the recycled 40S subunits to prevent their re-engagement with the 60S subunits (Jackson, Hellen and Pestova, 2010).

To mark the onset of initiation, a ternary complex containing eIF2, Met-tRNA ${ }_{i}^{\text {Mt }}$ and GTP is formed. This complex subsequently binds to the small ribosomal subunit through the coordinated efforts of eIF1, eIF1A, and eIF3 to yield the $43 \mathrm{~S}$ pre-initiation complex (PIC) (Kapp and Lorsch, 2004). The 43S PIC is recruited to the 5' cap in aprocess coordinated by eIF3, poly-A-binding-protein (PABP) and the eIF4F complex, which is composed of eIF4E, eIF4A and eIF4G (Sonenberg and Hinnebusch, 2009). eIF4E is considered the obligatory cap-binding protein. eIF4A is a RNA helicase that couples the hydrolysis of ATP to the binding of RNA and duplex separation, which promotes the "activation" of mRNA via unwinding of 5' untranslated region (5'UTR) secondary structures. eIF4G is a scaffold protein that possesses binding domains that aid in the coordinated assembly of the PIC and its attachment to the template mRNA (López-Lastra, Rivas and Barría, 2005; López-Lastra et al., 2010). The interaction of eIF4G with the mRNA facilitates synchronization amongst the 5 ' cap, poly-A tail, and mRNA sequence to assemble into a stable, circular messenger ribonucleoprotein (mRNP). A "protein bridge" is then established between the mRNP and the 43S PIC to promote the attachment of the $43 \mathrm{~S}$ to the mRNA, with aid from eIF4A (Sonenberg and Hinnebusch, 2009).

Once assembled at the cap, the 5'UT R secondary structures are unwound and the energy-dependent 5' to 3' scanning of the mRNA begins, looking to identify an initiation 
codon in a favorable sequence context, known as the Kozak consensus sequence (Kapp and Lorsch, 2004). The ribosome halts when stable base-pairing between the anticodon of Met-tRNAi (CAU) and the initiator AUG in the P-site of the 40S subunit occurs (Sonenberg and Hinnebusch, 2009). The recognition of the initiation codon, promotedby eIF1 and eIF1A, induces a conformational change that leads to the displacement of eIF1 and causes hydrolysis of the eIF2-bound GT P within the ternary complex to a GDP-bound state by eIF5, a GT Pase-activating protein (López-Lastra, Rivas and Barría, 2005). The subsequent joining of the large ribosomal subunit is mediated by eIF5B and catalyzes the mass dissociation of initiation factors eIF1, eIF3 and any residual eIF2-GDP from the complex (Kapp and Lorsch, 2004; López-Lastra, Rivas and Barría, 2005). Dissociation of eIF1A and eIF5B then occurs following the hydrolysis of eIF5B-bound GT P, which frees up the A-site and results in the formation of an elongation-competent $80 \mathrm{~S}$ ribosome (López-Lastra, Rivas and Barría, 2005; Sonenberg and Hinnebusch, 2009; Jackson, Hellen and Pestova, 2010). This event is thought to mark the conclusion of translation initiation, though supplementary steps may be required prior to the establishment of a fully active and peptide-bond forming complex (Kapp and Lorsch, 2004). 


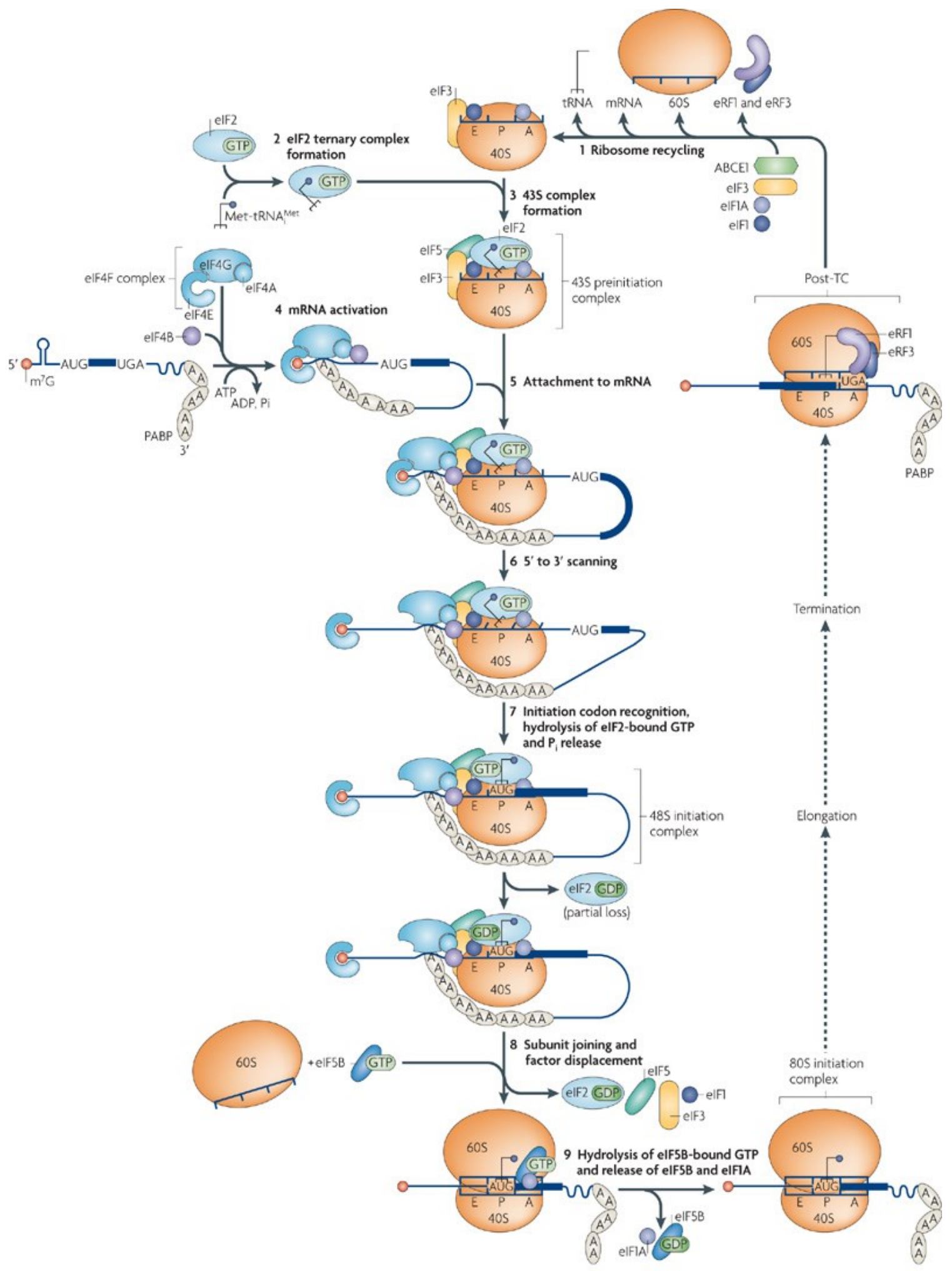

Figure 2. Schematic diagram of cap-dependent translation initiation in eukaryotes. Details of process described in the text. Reproduced with permission from (Jackson, Hellen and Pestova, 2010). 


\subsubsection{Eukaryotic Cap-Independent Mechanis m of Trans lation Initiation}

While most mRNAs are initiated via the canonical cap-dependent mechanism of translation initiation, there are mechanisms that do not rely on the 5' cap to initiate translation. These mechanisms tend to be employed to overcome limitationsenactedby the mRNA and cell state, and including leaky scanning, ribosome shunting, terminationreinitiation and internal initiation.

\subsubsection{Leaky Scanning}

The selection of a suitable start codon during the initiation of translation relies heavily on two parameters; the position effect and the Kozak consensus sequence. The position effect refers to how the first AUG encountered is favored as the initiation codon (Kozak, 2002). This has been demonstrated; 1) when a mutation generates an AUG upstream of the authentic start codon and initiation is consequently observed to shift to this upstream site, and 2) the removal of the authentic start codon prompts initiation to shift downstream to the subsequent AUG encountered(Bergenhem et al., 1992; Mével-Ninio et al., 1996; Kozak, 2002).

While an important parameter, codon positioning does not always dictate initiation preference; the Kozak consensus sequence also exerts influence on the decision. The Kozak consensus sequence specifies that the nucleotide composition surrounding the initiation codon, experimentally verified as 5'-GCCGCC(A/G)CCAUGG-3' in vertebrates, will considerably impact the affinity of the ribosome for the AUGin question (Kozak, 1987). Thus, if the primary AUG is presented in an unfavorable context, such as lacking a purine at the third base upstream of the $\mathrm{AUG}(-\mathbf{- 3})$ as well as a Gone base downstream of the AUG 
$(+1)$, then the ribosome might bypass it and continue scanning further downstream for a more suitable start codon (López-Lastra et al., 2010). This results in initiation from both AUGcodons (although, not equally) present in these mRNA sequences (Ryabova, Pooggin and Hohn, 2006). Accordingly, leaky scanning can allow for an mRNA transcript to encode for two different proteins, such that; a) dissimilar proteins are produced if the start codons are out of frame, or b) isoforms with varying $\mathrm{N}$-termini lengths are generated should the start codon remain in-frame (Kozak, 1991a).

Importantly, while leaky scanning predominantly occurs due to a weak consensus sequence, it may also occur if the primary AUGcodon is positioned too proximally, within a range of 12 nucleotides, of the 5' cap (Kozak, 1991b). Leaky scanning is a mechanism commonly exploited by mammalian viruses, including but not limited to; Human papillomavirus (HPV), Influenza A and Influenza B (Stacey et al., 2000; Jaafar and Kieft, 2019).

\subsubsection{Ribosome Shunting}

Ribosome shunting is a unique process that incorporates aspects of cap-dependent initiation with internal initiation. Ribosomes are recruited to the mRNA in a cap-dependent approach but scanning through the 5' UTR occurs in a discontinuous manner. The $40 \mathrm{~S}$ ribosome will bypass complex secondary structures within the leader regions by being shunted from the upstream donor site to a downstream acceptor site as to initiate translation (Hertz et al., 2013). This mechanism allows for the avoidance of inhibitory elements, which simultaneously maintains the ease of scanning and preserves the integrity of potentialRNA regulatory elements (Ryabova, Pooggin and Hohn, 2006; López-Lastra et al., 2010). 
Ribosome shunting was first discovered in 1993, and is now most extensively documented in Cauliflower mosaic virus (CaMV), which was then followed by Rice tungro bacilliform virus (RTBV) in 1996, both plant pararetroviruses (Ryabova, Pooggin and Hohn, 2006). Both viruses possess a leader sequence composed of multiple short open reading frames (sORFs) followed by a stable hairpin, which obstructs the option for linear scanning to occur. In both cases, the 5'-proximal sORF within the leader is translated prior to encountering the hairpin structure, which then forces the ribosome to shunt to a downstream portion of the mRNA sequence (pairing ribosome shunting and reinitiation, which is mentioned below). While identified in plant viruses, ribosomal shunting also occurs in animal viruses and in cellular mRNAs (Pooggin and Ryabova, 2018).

\subsubsection{Reinitiation}

Reinitiation is a form of internal initiation that allows for the expression ofmultiple cistrons from a single mRNA. This occurs when ribosomes have translated a short upstream open reading frame (uORF) and remain associated with the mRNA, with initiation resuming at a downstream start codon within vicinity of the stop codon (LópezLastra et al., 2010; Zinoviev, Hellen and Pestova, 2015). The efficiency of reinitiation varies depending on several features, including the following three. Firstly, reinitiation efficiency is increased as the intercistonic length is increased, but only in the absence of complex secondary structures, as it is believed that the ribosomes reacquire lost initiation factors prior to reinitiation (Kozak, 1987). Secondly, the length of the uORF directly impacts reinitiation efficiency, with shorter uORFs ( $<30$ codons) increasing the rate of occurrence (Ryabova, Pooggin and Hohn, 2006). Thirdly, composition of the uORF 
impacts reinitiation efficiency. For example, the presence of complex secondary structures within the uORF, such as a pseudoknot, would decrease efficiency (Somogyi et al., 1993).

Overall, the regulatory control over the process of reinitiation is quite complex and presently not well understood, with a multitude of additional influences further impacting the frequency of occurrence (Pooggin, Hohn and Fütterer, 2000).

\subsubsection{Internal Ribosome Entry Site}

Prior to discovery of the various cap-independent initiation mechanisms presented in the sections above, the cap-dependent method was thought to be the only means by which translation initiation could occur within eukaryotes. This idea was challenged in the late 1980s, when viral gene expression studies led to the identification of an alternative mechanism of initiation independent of the 5' cap structure (Pelletier and Sonenberg, 1988). This mechanism, termed Internal Ribosome Entry Site (IRES), allowed for a complex RNA secondary structure, commonly in the 5'UTR, to directly recruit ribosomes within vicinity of the start codon (Komar and Hatzoglou, 2011a). While this mechanism was originally characterized in picornaviruses, additional viral families have been observed to utilize this form of internal initiation, and it has also been recognized in a variety of cellular mRNAs (López-Lastra, Rivas and Barría, 2005).

IRES-mediated translation is a robust process that is commonly exploited by viruses to ensure that the viral mRNA is efficiently translated while the translation of host mRNAs is restricted (López-Lastra et al., 2010). While this primary goal is the same amongst all viruses, the activation of these viral IRESs is diverse. Variances include the structural organization of the IRES element, the requirements of canonical initiation 
factors, the requirements of additional, non-canonical proteins (dubbed IRES trans-acting factors, or IT AFS) and the recruitment strategy of ribosomal subunits to the IRES (Kieft, 2008; López-Lastra et al., 2010; Mailliot and Martin, 2018). Because of the significance these IRESs have had in viral infection propagation, substantial effort has been made to understand the intricacies of this process. This has ultimately led to classification of the viral IRESs into four different classes, constructed based on recognized structural and functional characteristics (Kieft, 2008; Mailliot \& Martin, 2018). There is a trendwhereby IRES elements with more sophisticated structural configurations tend to require fewer additional factors to initiate translation, and as the IRES becomes less structured, more requirements (such as eIFs and IT AFs) become requisite (Figure 3) (Kieft, 2008).

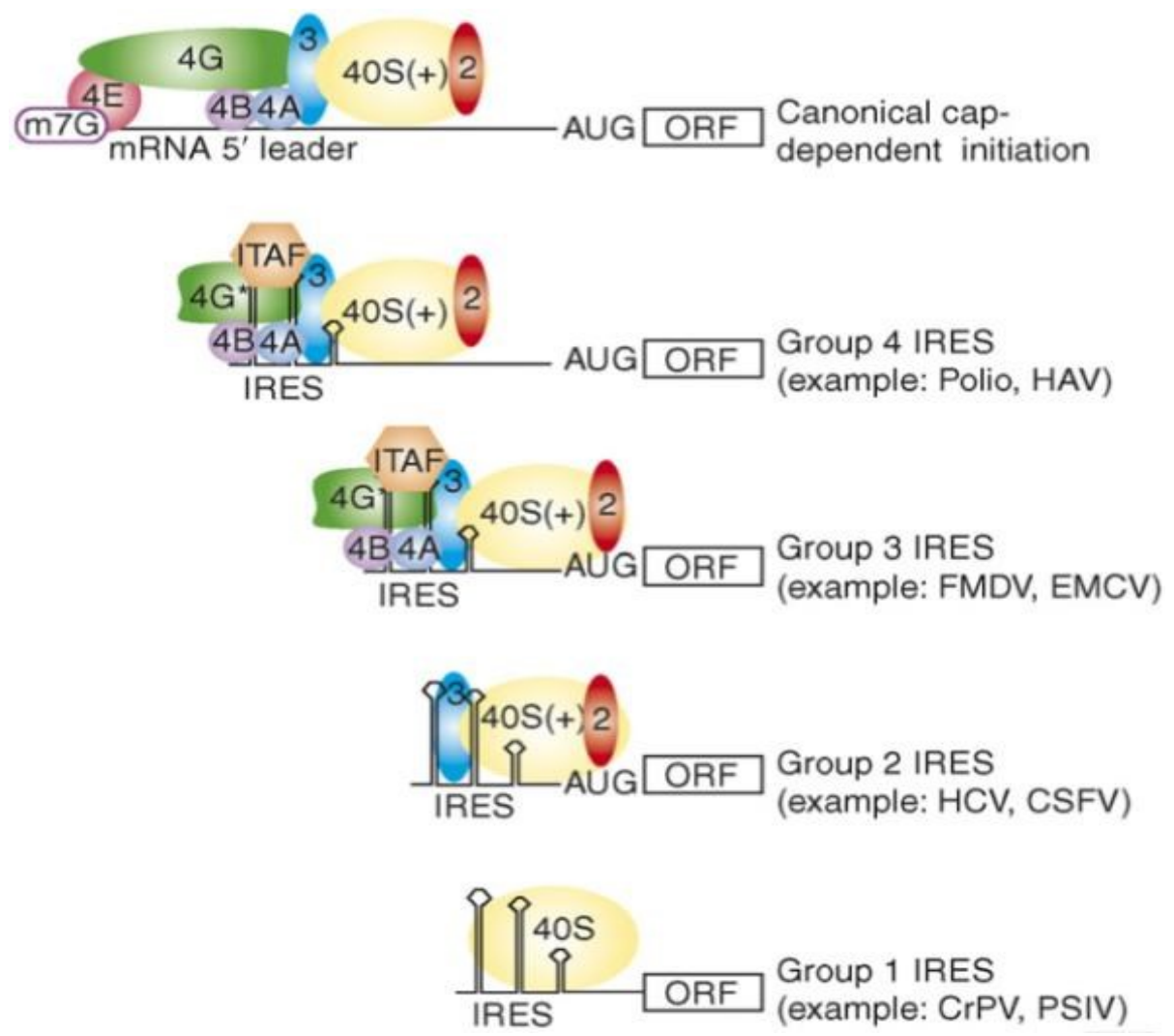

Figure 3. Visual depiction of viral IRES groupings and their requirements to initiate translation, compared with canonical cap-dependent translation initiation. As IRES elements become less structured, there is an increase in the requirements for eIFs and ITAFs to initiate translation. Reproduced with permission from (Kieft, 2008). 
The first viral IRES grouping, Class 4, refers to IRESs that form highly intricate secondary and tertiary RNA structures that are capable of directly binding to the ribosome and assembling an $80 \mathrm{~S}$ complex independent of the scanning process, protein factors or Met-tRNA $_{i}{ }^{\text {Met }}$ (Kieft, 2008; Lozano and Martínez-Salas, 2015; Martinez-Salas et al.,2018; Jaafar and Kieft, 2019). This group is composed of conserved IRESs located in the intergenic region (IGR) between open-reading frames (ORFs) in the viral RNA of singlestranded positive-sense Dicistroviridae viruses, such as Isreali acute paralysis virus (IAPV), Plautia stali intestinal virus (PSIV) and Cricket paralysis virus (CrPV) (Mailliot and Martin, 2018). These IGR IRESs adopt a highly compact 3-D structure composed of three pseudoknots (PK) (PKI, II and III), two stem-loops (SL) (SL IV and V), and two independently folding domains (the larger domain possessing regions I and II, and the separate smaller domain III) (Kieft, 2008). The larger domain possesses affinity for the ribosome, as its two stem loops directly contact the small subunit and induce a conformation change of the 40 S subunit. The smaller Domain III possesses PKI, which structurally mimics Met-tRNAi and allows for an imitation tRNA-mRNA interaction to occur within the ribosomal A-site (Jaafar \& Kieft, 2019; Kieft, 2008). Thus, PKI docks into the A-site, sets the appropriate ORF and initiates translation via pseudotranslocation of PKI into the P-site and a non-AUG start codon into the A-site of the 40S ribosomal subunit (Jan, Kinzy and Sarnow, 2003; Kieft, 2008; Johnson et al., 2017; Jaafar and Kieft, 2019).

The second viral IRES grouping, Class 3, refers to IRES elements present at the 5' end of the viral transcript that directly bind to the $40 \mathrm{~S}$ subunit and only utilize a limited subset of canonical initiation factors, as well as Met-tRNAi, to initiate translation (Pestova 
et al., 2001). This IRES grouping possesses a large, extended general structure, with subdomains of rigid segments interposed by adaptable bridges (Filbin and Kieft, 2009; Mailliot and Martin, 2018). This group consists of members from the family Flaviviridae, including the pestiviruses Classical swine fever virus (CSFV) and Bovine viral diarrhea virus (BVDV), and hepaciviruses such as Hepatitis C virus (HCV). The IRES of HCV is exceptionally well documented and its mechanism is reasonably elucidated, and thus will be expanded on.

The 5' UT R of HCV contains four domains (I-IV). Domains I and II are involved in viral replication and the IRES element bridges domains II and III. Domain IV, which is present in HCVbut absent from other members of this Class (such as CSFV), contains the AUG start codon within a hairpin structure that is protracted upon IRES presence within the mRNA conduit (Mailliot and Martin, 2018). Mechanistically, the HCV IRES binds to the $40 \mathrm{~S}$ ribosome in a multi-domain interaction, altering the ribosome conformation and creating a 40S-IRES PIC (Kieft et al., 2001). After 40S ribosomal subunit recruitment, the HCV IRES domain III stably interacts with eIF3, which promotes the recruitment of the ternary complex to the 40S-IRESPIC, and results in the formation of a 48SPIC with the AUG codon present in the ribosomal P-site, without scanning (Pestova et al., 1998; Mailliot and Martin, 2018; Jaafar and Kieft, 2019). The 48S PIC then recruits the 60S subunit, and via subsequent GTP hydrolysis and eIF2 release, yields an 80 S ribosome properly positioned at the start codon (Lozano and Martínez-Salas, 2015). Thismechanism of initiation, in which $40 \mathrm{~S}$ recruitment occurs without the requirement of initiation factors, has been cited to be analogous to prokaryotic translation, with the IRES acting as a 
functional equivalent to the Shine-Dalgarno sequence (SD) (Gray and Wickens, 1998; Jackson, 2005).

The third and fourth viral IRES groupings, Class 1 and 2, refer to IRESs that require the use of some canonical initiation factors, ITAFs and Met-tRNAi, and are used by members of the Picornaviridae family. Class 1 members include the enterovirus Poliovirus (PV) and the apthovirus Rhinovirus (RV), whereas Class 2 include members such as the cardiovirus Encephalomyocarditis virus (EMCV) and apthovirus Foot-and-Mouth disease virus (FMDV) (Mailliot and Martin, 2018). These IRESs do not form compact structures, but rather maintain extended and flexible conformations, which consequently makes it problematic to produce high-resolution 3-D models of the elements (Filbin and Kieft, 2009). These two groupings of viral IRESs are similar to one another based on their: presence in the 5' UTR of the viral transcript, sequence length, inability to directly bindto the $40 \mathrm{~S}$ subunit, requirement of the near-complete set of canonical initiation factors(which does not include eIF4E), presence of a pyrimidine-rich tract located at the 3 ' end of the IRES and recruitment of ribosomes following the repression of host cap-dependent translation (Jackson, 2005; Kieft, 2008; Mailliot \& Martin, 2018). The IRES of PV for Class 1, and IRESs of EMCV and FMDV for Class 2 will be used as examples.

The 5' UTR of PV contains six domains (I-VI), with domain I essential for viral replication and the IRES composed of domains II-VI (Mailliot and Martin, 2018). The PV IRES possesses a cryptic AUG necessary for ribosome recruitment, but the ribosome will scan 160 nucleotides further downstream to initiate translation at a secondary AUGcodon (Jackson, 2005; Lozano and Martínez-Salas, 2015; Martinez-Salas et al., 2018). It isknown that translation initiation via Class 1 IRESs require all eIFs (minus eIF4E) and the presence 
of IT AFs, such as poly(C)-binding protein 2 (PCBP-2) and polypyrimidine-tract binding protein (PTB), but the mechanism has yet to be fully elucidated, with little identified regarding its requirements for canonical factors or their responsibilities (De Breyne et al., 2009; Mailliot and Martin, 2018).

The 5' UTR of EMCV and FMDV contain five domains (I-V), with domain I required for viral replication, and the IRES composed of domains II-V (Mailliot and Martin, 2018). Both EMCV and FMDV possess two in-frame AUG codons and the ribosomes bind to them directly, without scanning (Pestova et al., 2001; Jackson, 2005). With the EMCV IRES, only the second AUGis used as a start codon, present downstream of the beginning of the pyrimidine tract, whereas both AUGs are utilized to initiate translation with the FMDV IRES (Jackson, 2005; Mailliot and Martin, 2018). Also incongruent, EMCV and FMDV have dissimilar IT AF requirements, despite their relatedness (Pestova et al., 2001; Jackson, 2005; Martinez-Salas et al., 2018). In the examples presented of both Classes, a domain is present (domain Vand domain IV, for the PV and EMCV/FMDV IRESs, respectively) that provides a binding site for eIF4G. The binding of eIF4G causes the recruitment of eIF4A, which further enhances the eIF4G-IRES interaction and alters the IRES conformation as to increase its binding affinity to the $43 \mathrm{~S}$ complex (De Breyne et al., 2009). Thus, a shared fundamental mechanism underlies initiation for the unrelated Class 1 and Class 2 IRESs, despite lack of sequence and structural homology (De Breyne et al., 2009).

Unlike picornavirus RNA genomes, which are uncapped and necessitate the use of IRESs, it is assumed that all cellular mRNAs are capped and capable of undergoing cap dependent initiation (Pestova et al., 2001; Komar and Hatzoglou, 2011b). Despite this 
detail, there are many cellular mRNAs that have been identified to possess IRES elements, with both mechanisms operating on the same message (Komar and Hatzoglou, 2011b). It is thought that these cellular IRESs possess two major physiological functions; 1) allow for the translation of mRNAs with complex 5' UTRs under normal physiological conditions, and 2) maintain translation of mRNAs under various (patho)physiological stress conditions (Komar and Hatzoglou, 2011b; Pichon et al., 2012; Moteshareie et al., 2018). As expected, many mRNAs identified to possess an IRES element function to maintain cell homeostasis and play a regulatory role in cell-fate decisions, including transcription factors (HIF-1 $\alpha$ ), oncogenes (c-myc), cell cycle control genes (Cyclin D1) and apoptotic genes (Apaf-1 and Bcl-2) (Stoneley et al., 1998; Coldwell et al., 2000;Lang, Kappel and Goodall, 2002; Sherrill et al., 2004; Shi et al., 2005; Pichon et al., 2012).

While the structure and mechanism of action is well characterized regarding many viral IRESs, the specifics surrounding cellular IRESs are difficult to elucidate. This is attributable to the fact that cellular IRESs are highly diverse, with no conserved sequence or secondary structure unifying them (Komar and Hatzoglou, $2011 \mathrm{a}$; Jackson, 2013). It is important to note that IRES elements are completely dependent on their structural integrity, with the presence of any alterations (such as small indels or point mutations) within the IRES element significantly altering its activity. It has also been suggested that the folding of IRES into its tertiary structure is a dynamic process, implying that specific environmental conditions can cause modification of the IRES structure and consequently alter its translational capabilities (López-Lastra, Rivas and Barría, 2005). 


\subsubsection{Prokaryotic Mechanism of Translation Initiation}

In eukaryotes, translation initiation is a highly complex process relying on mRNAprotein and protein-protein interactions (López-Lastra, Rivas and Barría, 2005). However, translation initiation in prokaryotes is a much simpler process, one that consists of the recruitment of a complex of ribosome-initiator tRNA to the initiation codon of a nascent mRNA strand via direct interaction of the mRNA with the rRNA. Prokaryotic translation initiation only requires the large (50S) and small (30S) ribosomal subunits, mRNA, initiator tRNA, three initiation factors (IF1, IF2 and IF3), and GT P as the energy source (Figure 4) (Laursen et al., 2005; Gualerzi and Pon, 2015). The three IFs determine the fidelity and kinetics of the initiation process, each bound as a single copy to critical sites on the 30S subunit (Milón and Rodnina, 2012; Gualerzi and Pon, 2015).

Comparable to the recycling step within eukaryotic initiation, bacterial initiation is activated when subunits from the previous rounds of translation are dissociated and recycled with help from initiation factors. The dissociation process is promoted by IF3 binding to the $30 \mathrm{~S}$ subunit, in tandem with IF1. IF1 binds to the A-site of the 30 S subunit to direct the initiator tRNA into the P-site, which will further stimulate IF3 activity and further promote the dissociation of subunits (Laursen et al., 2005). Marking the onset of the initiation pathway, all three IFs, mRNA, and fMet-tRNA ${ }^{\mathrm{AMet}}$ associate with the $30 \mathrm{~S}$ ribosomal subunit, leading to the formation of the 30S PIC (Simonetti et al., 2009). The 30S PIC associates with the mRNA via a ribosome binding site (RBS), the SD sequence, and causes the initiation codon and the initiator tRNA to be adjusted into the P-site of the ribosome by the IFs (Shine and Dalgarno, 1974; Laursen et al., 2005). Once the decoding process has occurred, the 30S PIC is joined by the large subunit to yield the 70S initiation 
complex. This action triggers GT P hydrolysis of IF2, which confirms the start site and forces the dissociation of superfluous complexes (Simonetti et al., 2009). This event is thought to mark the conclusion of translation initiation and allows the translation machinery to proceed to the elongation phase.

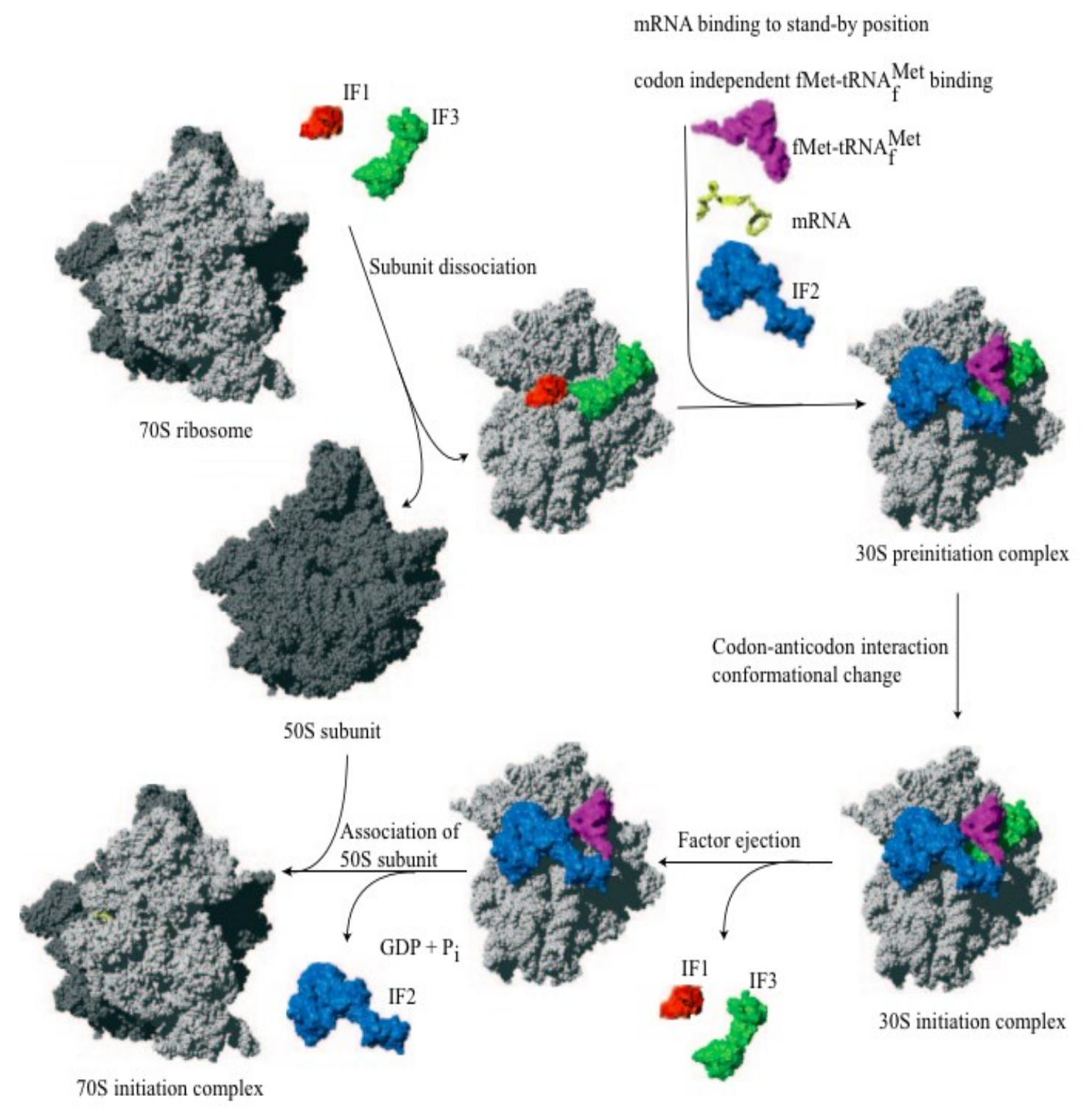

Figure 4. Schematic diagram of prokaryotic translation initiation. The colour coding is as follows: 30S ribosomal subunits (light grey), 50S ribosomal subunits (dark grey), IF1 (red), IF2 (blue), and IF3 (green), mRNA (yellow), and fMet-tRNA ${ }^{\text {fMet }}$ (magenta). Details of the process described in the text. Reproduced with permission from (Laursen et al., 2005). 


\subsubsection{Shine-Dalgarno and Enhancer Seque nces}

The SD sequence is a ribosome binding sequence present in bacterial and archaeal mRNA. This is a purine-rich consensus sequence, AGGAGGU (in E. coli), that is present several nucleotides upstream of the AUG start codon and forms base-pairing interactions with a pyrimidine-rich tract, ACCUCCUUA, present at the 3' end of the 16SrRNA(known as the anti-SD) in the 30S subunit (Shine and Dalgarno, 1974; Steitz and Jakest, 1975). The SD helix duplexes with the anti-SD and correspondingly fits within a cleft between the subunit head and the back of the platform of the 16SrRNA. This interaction helpsto recruit the 30 S ribosome to the mRNA, and along with a spacer region (optimally 4-9 nucleotides in E. coli), sets the reading frame and allows for correct placement of the start codon within the P-site of the 30S subunit (Yusupova et al., 2001; Kaminishi et al., 2007; Gualerzi and Pon, 2015). It is important to note that the SD sequence is loosely conserved and can vary in length, along with the spacing between the TIR and itself, with deviations having a discernible effect on translation efficiency (Shultzaberger et al., 2001; Vimberg et al., 2007).

While the binding of the SD consensus sequence is a critical step in the initiation of prokaryotic translation, there are many bacterial mRNAs in which SD sequences are absent and yet, are still efficiently translated (Gualerzi and Pon, 2015). Other sequence elements have been identified that are significant for regulating translation initiation efficiency, one of which is epsilon. The epsilon sequence element (UUAACUUUA) is a translational enhancer that exhibits complementarity to a portion of helix 17 (nucleotides 458-466) of the 16S rRNA (A Golshani et al., 2000; Ashkan Golshani et al., 2000; O'Connor, 2001). While originally identified in the 5'UTR of the phage T7 gene 10 
mRNA, it was observed that upstream placement of the element enhanced the translational activity of several genes, including a lacZ reporter construct, proposed by base-pairing to its complementary region (Olins and Rangwala, 1989). It was identified by Golshani et al. that not only did the initial epsilon element enhance translation of mRNAs containing an SD sequence, but constructs containing an extended epsilon element could also efficiently direct translation initiation in the absence of an SD sequence (Golshani et al., 1997, 2004).

\subsection{Proposed Similarities between Prokaryotic \& Eukaryotic Translation Initiation}

In 2015, Colussi et al. published an article in Nature which reported that a eukaryotic IRES, the PSIV IGR IRES, can initiate in vivo translation in prokaryotes, with its activity appearing to be independent of an SD-based method of translation initiation (Colussi et al., 2015). To come to this conclusion, they had employed a dual-luciferase expression vector in which the structured PSIV IGR IRES was used to drive expression of Firefly luciferase (FLuc), the experimental reporter, while an SD and enhancer sequence expressed Renilla luciferase (RLuc), an internal control. Their resulting normalized expression data confirmed that the PSIV IGR IRES was functional and can indeed initiate translation within E. coli. Colussi et al. had also observed that when the structural integrity of the IRES was compromised, via disruption of the PKs within the st ructure or use of the isolated Domain III, IRES functionality was reduced. This indicated that initiation via the PSIV IGR IRES in bacteria depends on the structural integrity of the compact RNA element, like that in eukaryotes. 
They had also elucidated the crystal structure of the IRES element associated with the $70 \mathrm{~S}$ ribosome complex to a $3.8 \AA$ resolution. The results indicate that the IRES can bind within the tRNA binding sites of bacterial ribosomes, albeit differently than that of eukaryotes. The IRES element appears to form weak and temporary interactions with the bacterial ribosomes, but still remains functional and allows for internal initiation to occur. The proposed mechanism of prokaryotic translation initiation is also dissimilar relativeto that observed in eukaryotes. In the prokaryotic mechanism, the ribosome repositionsfrom the IRES to the downstream FLuc AUGto initiate bacterial translation, as opposed to the direct initiation at a non-AUG start codon that is typical of eukaryotic functioning. Importantly, Colussi et al. similarly attempted to initiate prokaryotic translation using the IRES of CSFV. This IRES element produced negligible expression levels of FLuc, which illustrated the specificity towards the PSIV IGR IRES. These results, when taken together, appear to suggest that there may be a conserved approach of some features of initiation, based on the structure of this initiation element, between prokaryotes and eukaryotes.

\subsection{Focus and Objectives}

In both prokaryotes and eukaryotes, translation is initiated via the recruitment of ribosomal subunits to an mRNA transcript in a sequential and multi-step procedure. In general, eukaryotes recruit the small ribosomal subunit to the 5' cap via protein-protein and protein-RNA interactions, followed by scanning of the mRNA sequence. The initiation codon in a favorable context is identified and the 60S subunit joins and completes the assemblage, which is then ready to begin polypeptide synthesis. In prokaryotes, the small ribosomal subunit is directly recruited to the vicinity of the initiation codon. Via a base- 
pairing interaction between the $16 \mathrm{~S}$ rRNA and the SD sequence on the mRNA, the initiation codon is precisely positioned into the ribosomal $\mathrm{P}$-site, equipping the complex for elongation. These pathways have become so divergent over the millennia, eukaryotes are unable to utilize the SD sequence and prokaryotes are incapable of recognizing the 5' cap structure of eukaryotic mRNAs (Colussi et al., 2015). Even with the existence of alternative initiation mechanisms in both domains of life, no universal mechanism hadbeen observed to exist that can overlap between the two, on similar message types.

Conversely, the 2015 Colussi et al. article suggested that the PSIV IGR IRES can initiate translation within E. coli independent of an SD-based mechanism. It appears that to be functional in a prokaryote, the IRES relies on its 3-D structure and the preservation of its structural integrity to initiate translation, like that in eukaryotes. This wouldinsinuate that there is a conserved approach of initiation based on the structure of this element present between prokaryotes and eukaryotes.

The objective of this thesis is two-fold; 1) to investigate whether the initiation capabilities of the PSIV IGR IRES in prokaryotes is a unique characteristic, or whether other IRES elements, or other complex mRNA structures, can also mediate prokaryotic translation initiation, and 2) to elucidate any potential distinguishing characteristics that allow the PSIVIGR IRES to initiate translation in prokaryotes. To address these objectives, the methodology included the following two key features, all of which were carried out using the same dual-luciferase reporter construct as that of Colussi et al. (2015).

Firstly, to deduce whether the prokaryotic initiation capabilities of the PSIVIGR IRES is a unique characteristic, or whether it is a common property amongst IRES elements, a variety of IRES (viral and cellular) and structured RNA elements, were cloned 
between the two luciferases of the dicistronic construct. The luciferase expression data presented by Colussi et al. was confirmed via experimental replication (using the constructs received from Colussi et al.) for PSIV, used as a positive control, and CSFV, used as a negative control. Following protein expression mediated by the various IRES and structured RNA elements, it appears that the ability of the PSIV IGR IRES to initiate prokaryotic translation is a distinct property that is not shared among the other IRES elements tested.

Secondly, to determine whether the PSIV IGR IRES possesses any specific differentiating characteristics, the sequence of the IRES element was mutated (several times) and their ability to initiate translation was evaluated by expression analysis. During these experiments, focus was placed on the initiating capabilities of the PSIV Domain III, as it was previously reported that this domain can bind to the $70 \mathrm{~S}$ ribosomes through its proper positioning in the decoding groove of the small ribosomal subunit (Zhu et al., 2011; Colussi et al., 2015). Additionally, it was observed that relative to the luciferase expression data for the Full-Length Domain III presented by Colussi et al., our experimentally replicated Full-Length Domain III construct expressed luciferase at considerably lower rates.

The first part of the second objective involved alterations to the Domain III sequence by truncation of the structure via 5' end nucleotide deletions. The truncation of Domain III altered the structural integrity of the IRES and destroyed a PK necessary for eukaryotic translation initiation. The second part of the second objective involved the introduction of four-point mutations within two regions of interest in the truncatedelement. The two specified regions of interest had been identified to possess complementarity 
between itself and the $16 \mathrm{~S}$ rRNA, and the point mutations were introduced as to disrupt any base-pairing capabilities. The Truncated Domain III construct was observed to have an increased ability to initiate prokaryotic translation relative to the Full-Length Domain III, whereas the mutated elements were observed to have a severely reduced ability to initiate prokaryotic translation relative to the Truncated Domain III. Together, these results indicate that the 3-D structure of the IRES appears to have less significance in prokaryotes than previously suggested and that the complementary regions within Domain III might bear some responsibility in the ability of this IRES to initiate prokaryotic translation. 


\section{Journal Article}

\subsection{Introduction}

Translation is a universally conserved step within the process of gene expression. Of the steps that comprise translation, translation initiation is the most phylogenetically diverse (Laursen et al., 2005). While both prokaryotes and eukaryotes initiate translation via the recruitment of ribosomal subunits to an mRNA transcript in a sequential and multistep procedure, the details of their execution are dissimilar (Laursen et al., 2005; Benelli and Londei, 2011). In prokaryotes, the small ribosomal subunit is directly recruited to the vicinity of the initiation codon, using only three initiation factors (Gualerzi andPon, 2015). Through a RNA base-pairing interaction between the 16S rRNA and the Shine-Dalgarno (SD) sequence on the mRNA, the initiation codon is precisely positioned into the ribosomal P-site, equipping the complex for elongation (Laursen et al., 2005). Alternatively, eukaryotes predominantly recruit the small ribosomal subunit to the 5' cap via proteinprotein and protein-RNA interactions, with the assistance of over a dozen initiation factors (López-Lastra, Rivas and Barría, 2005). The directional scanning of the mRNA sequence occurs to identify the initiation codon in a favorable context and the $60 \mathrm{~S}$ subunit joins to complete the assemblage, which is then ready to begin polypeptide synthesis (Sonenberg and Hinnebusch, 2009). Prokaryotic and eukaryotic translation initiation pathways have become so divergent over the millennia, that bacteria and eukaryotes are unable to recognize or utilize the translational signals present in the other's mRNA sequence (Colusi et al., 2015). Thus, despite the existence of alternative initiation mechanisms in both domains of life, no universal mechanism had been observed to exist that can overlap between the two, on similar message types. 
Importantly, eukaryotes can also initiate translation through alternative initiation mechanisms, exemplified by the use of an Internal Ribosome Entry Site (IRES). IRES allows for a complex RNA secondary structure to directly recruit ribosomes within vicinity of the start codon, operating independently of the 5' cap structure (Komar and Hatzoglou, 2011a). This mechanism has been found to be used by both cellular mRNAs, to maintain cell homeostasis and play a regulatory role in cell-fate decisions, as well as viruses, to ensure that the viral mRNA is efficiently translated while the translation of host mRNAs is restricted (López-Lastra et al., 2010; Komar and Hatzoglou, 2011 a). The structure and mechanisms of viral IRESs are reasonably well-characterized compared to cellular IRESs.

The primary goal of IRES functioning is the same amongst all viruses, but the activation and presentation of these viral IRESs is quite diverse. Viral IRESs can differ with regards to their structural organization, requirements of canonical initiation factors, requirements of additional, non-canonical proteins (dubbed IRES trans-acting factors, or IT AFS) and recruitment strategy of ribosomal subunits (Kieft, 2008; López-Lastra et al., 2010; Mailliot and Martin, 2018). Nevertheless, because of the significance these IRESs have had in viral infection propagation, substantial effort has been made to understand the intricacies of this process. This has ultimately led to classification of the viral IRESs, constructed based on recognized structural and functional characteristics (Kieft, 2008; Mailliot \& Martin, 2018).

Class 1 of the viral IRESs is of particular importance, as it possesses the most streamlined mechanism of ribosomal manipulation known. This Class is composed of IRESs found in the intergenic region (IGR) between open-reading frames (ORFs) in the viral RNA of single-stranded positive-sense Dicistroviridae viruses, and include those 
found in Plautia stali intestinal virus (PSIV) and Cricket paralysis virus (CrPV) (Mailliot and Martin, 2018). These IGR IRESs adopt a highly compact 3-D RNA structure that is capable of directly binding to the ribosome and assembling an elongation-competent $80 \mathrm{~S}$ ribosome independent of the scanning process, protein factors or Met-tRNA ${ }_{i}{ }^{\text {Met }}$, by mimicking the tRNA-mRNA interaction within the decoding site of the small ribosomal subunit and inducing a pseudotranslocation event (Kieft, 2008; Lozano and MartínezSalas, 2015; Martinez-Salas et al., 2018; Jaafar and Kieft, 2019).

It appears that one member of this Class is not only capable of controlling eukaryotic ribosomes, but also prokaryotic ribosomes. In 2015, Colussi et al. reported that the PSIV IGR IRES can initiate in vivo prokaryotic translation (Colussi et al., 2015). They concluded that the activity of the PSIV IGR IRES is independent of an SD-based method of translation initiation. Rather, the IRES can directly manipulate the phylogenetically conserved core of the ribosome by binding to the ribosomal P-site using tRNA mimicry, like that in eukaryotes, to initiate prokaryotic translation. Importantly, this IRES was deduced to rely on its 3-D structure and the preservation of its structural integrity to initiate translation, also as in eukaryotes. These results would provide evidence towards a conserved approach of initiation based on the structure of this element in both prokaryotes and eukaryotes.

This discovery led us to study different aspects surrounding the ability of the PSIV IGR IRES to initiate prokaryotic translation. Firstly, we sought to deduce whether the initiation capabilities of the PSIV IGR IRES in prokaryotes is a unique characteristic, or whether other IRESs, or other complex RNA structures, can also mediate prokaryotic translation initiation. Secondly, we attempted to elucidate any potential distinguishing 
characteristics that allow the PSIV IGR IRES to initiate translation in prokaryotes. To address these objectives all the methodology was carried out using the dual-luciferase reporter construct of Colussi et al. (2015). Our results indicate that the ability of the PSIV IGR IRES to initiate prokaryotic translation appears to be a distinct property that is not shared between the other IRES elements studied. It also seems that the 3-D structure of this IRES has less significance than previously suggested and that the presence of complementary regions within the Domain III of the IRES might bear some responsibility in its ability to initiate prokaryotic translation. 


\subsection{Methods}

\subsubsection{Organization of Dicis tronic Reporter Plas mid}

The inducible expression vector utilized was a modified pET30a (Novagen) plasmid, created and provided by the lab of Jeffery Kieft. This plasmid possesses DNA containing the PSIV IGR IRES (nucleotides 6000-6195) between the Renilla reniformis luciferase (Renilla, RLuc) and Photinus pyralis luciferase (Firefly, FLuc) genes (Figure 5). The RLuc gene is under the translational control of an SD and enhancer sequence, while the FLuc gene is expressed by the upstream IRES. The dicistronic genes are under the transcriptional control of a $\mathrm{T} 7$ promoter and the plasmid possesses a kanamycin resistance gene.

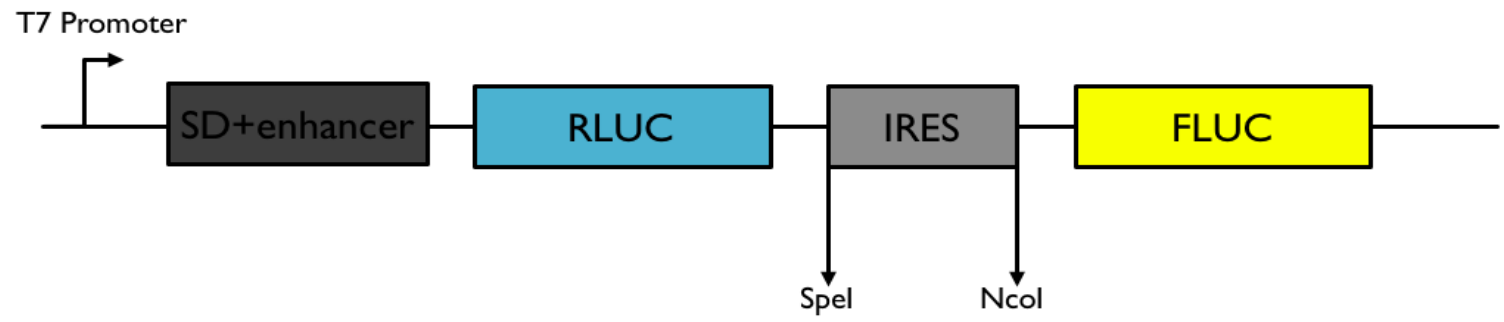

Figure 5. The dual-luciferase reporter construct within the modified pET30a vector utilized. This construct is under the transcriptional control of a T7 promoter. The internal control, RLuc, is under translational control of an SD and enhancer sequence. The experimental reporter, FLuc, is under the translational control of an IRES element. The IRES element is flanked by two restriction sites, SpeI and NcoI, for which cloning of various inserts was used. 


\subsubsection{Cloning of Dual-Lucife rase Cons tructs}

\subsubsection{Viral IRES, Cellular IRES, and Structured RNA Elements}

Each of the cellular IRES element inserts, from FLO8, GPR1, HAP4 and NCE102 were created via PCR amplification from the genomic DNA of Saccharomyces cerevisiae mating type $\alpha$. Each of the structured RNA element inserts were IRES elements from FLO8 and GPR1, which were PCR amplified from the genomic DNA of $S$. cerevisiae matingtype $\alpha$ and inserted into the restriction sites in reverse. Each of the additional two viral IRES element inserts, from FMDV and the IGR of CrPV, were created via PCR amplification from plasmids possessing the targeted IRES (FMDV IRES amplified from the pVIT ROHPV8 L1L2 plasmid, which was a gift from Richard Roden (Addgene plasmid \# 52598) (Kwak et al., 2014) and CrPV IRES amplified from pBgal/CAT plasmid provided by Dr. Martin Holcik). The PSIV IGR IRES and CSFV dual-luciferase constructs were kindly provided by the lab of Jeffery Kieft. The forward and reverse oligonucleotide primers (IDT) (Appendix 1. Table 2) possessed intact SpeI and NcoI restriction sites flanking the hybridization sequence. Amplified inserts were ligated into the Dual-LUC pET 30a vector between the SpeI and NcoI restriction sites using T4 DNA Ligase (NewEngland Biolabs).

\subsubsection{Mutated Domain III Elements}

Each of the PSIV Domain III inserts (Table 1) were synthesized by hybridizing complimentary forward and reverse oligonucleotide primers (IDT) possessing the desired sequence with specified mutations. The forward and reverse oligonucleotide primers(IDT) possessed destroyed SpeI and NcoI restriction sites flanking the hybridization sequence to be used as a confirmation method for cloning. These inserts were ligated into the Dual- 
LUC pET30a vector between the SpeI and NcoI restriction sites using T4 DNA Ligase (New England Biolabs).

Table 1. Sequence of mutated Domain III inserts. Strikethrough represents deleted nucleotides. Underline represents mutated bases. Bolded nucleotides represent complementary regions to the 16S rRNA. Respective destroyed restriction sites flank the 5' and $3^{\prime}$ ends of the presented sequences.

\begin{tabular}{cc}
\hline $\begin{array}{c}\text { Construct } \\
\text { Name }\end{array}$ & Sequence \\
\hline Full-Length & TGCTCGCTCAAACATTAAGTGGTGTTGTGCGAAAAGAATCTCACTTCAAGAAAA \\
Domain III & \\
\hline Truncated & TGCTCGCTCAAACATTAAGTGGTGTTGTGCGAAAAGAATCTCACTTCAAGAAAA \\
Domain III & \\
\hline ACTT & TGCTCGCTCAAACATTAAGTGGTGTTACTTGAAAAGAATCTCACTTCAA GAAAA \\
\hline CACT & TGCTCGCTCAAACATTAACACTTGTTGTGCGAAAAGAATCTCACTTCAA GAAAA \\
\hline
\end{tabular}

\subsubsection{Bacterial Cell Cultures}

Each of the cloned constructs were transformed into E. coli BL21 (DE3) competent cells (Invitrogen) using the heat shock method (Inoue, Nojima and Okayama, 1990). Positive transformants were confirmed via Sanger sequencing at the Ottawa Hospital Research Institute (OHRI).

\subsubsection{Luciferase Induction and Measurements}

An overnight starter culture was generated using transformed BL21 (DE3) cells grown in Luria Broth (LB) with kanamycin at $37^{\circ} \mathrm{C}$ with constant agitation. To begin the protein induction process, LB containing kanamycin was inoculated with the overnight starter culture and grown with agitation to an absorbance of 0.4-0.5 at $600 \mathrm{~nm}$ (measured 
on a Pharmacia Biotech Ultrospec 3000 spectrophotometer). The cultures were then induced with $1 \mathrm{mM}$ IPTG (BioShop) and allowed to grow for an additional 24 hours at $16^{\circ} \mathrm{C}$ prior to sample acquisition.

Extracted cells were pelleted by centrifugation and washed (1x PBS) prior to being re-pelleted and re-suspended in $250 \mu \mathrm{L} 1 \mathrm{X}$ Passive Lysis Buffer (PLB, Promega). Luciferase activity was monitored using the Dual-Luciferase ${ }^{\circledR}$ Reporter Assay System (Promega), per manufacturer's instructions. Luciferase activity was measured in an opaque white 96-well microplate (Nunc Plate, Thermo Fisher) using the Cytation 5 Cell Imaging Multi-Mode Reader (BioT ek Instruments) performed at $23 \pm 2{ }^{\circ} \mathrm{C}$. Experiments used the automatic dual-injection mode, which involves the automatic injection of assay buffersinto the wells containing cell lysates, and measured the resulting light intensity emitted overthe specified time-period (expressed as Relative Light Units; RLU's). PMT gain was set to a sensitivity of 80 and the optimal well reading height was determined to be $1 \mathrm{~mm}$ for the measurement of RLuc expression. PMT gain was set to a sensitivity of 150 and the optimal well reading height was determined to be $5 \mathrm{~mm}$ for the measurement of FLuc expression.

\subsubsection{Statis tical Analyses}

Figures of the cellular IRES and RNA element secondary structures were predicted and generated by RNAFold Server, in which the linear sequence of the element was entered and the minimum free energy (MFE) structure was generated using the algorithm of Zuker and Stiegler (Zuker and Stiegler, 1981; Hofacker, 2003).

IRES activity was deduced from the calculated and graphed normalized luciferase expression data at the 24-hour time point. Normalization of the dual-luciferase data, 
calculated as FLuc (experimental reporter) activity divided by RLuc (control reporter) activity, was done to correct for variability in growth patterns, induction, and potential differences in protein stability between individual cultures. Normalized $\Delta$ Fold Activity represents the fold change in normalized luciferase activity between the PSIV construct and other IRES constructs, set between 0 and 1, calculated as the average normalized luciferase activity of the specified IRES construct divided by the average normalized luciferase activity of the PSIV IRES construct. Averages for each IRES construct were generated from a minimum of four biological and three technical replicates. Error bars present within the graphs represent the standard errors of the average values for each construct.

To assess differences between the IRES activities of constructs, one-way analysis of variance (ANOVA) was applied followed by a post hoc Tukey Test. Statistical significance was assumed for values of $p<0.05$. 


\subsection{Results and Discussion}

Despite having a shared objective, the process of translation initiation is highly disparate between eukaryotes and prokaryotes, both mechanistically and with the initiation factors used. Both domains initiate translation via the recruitment of ribosomal subunitsto an mRNA transcript in a sequential and multi-step procedure. Although, eukaryotes generally utilize significantly more initiation factors than prokaryotes, and the process is more complex. Eukaryotic mRNAs possess a 5' cap structure to which the small ribosomal subunit is recruited to through protein-protein and protein-RNA interactions, followedby scanning of the 5'UTR as to identify an initiation codon in a favorable context. Once identified, the 70S subunit joins and completes the assemblage, which is then ready to begin polypeptide synthesis. However, prokaryotic mRNAs possess an SD sequence upstream of the initiation codon, to which the small ribosomal subunit is directly recruited to. Through an RNA base-pairing interaction between the 16SrRNA and the SD sequence on the mRNA, the initiation codon is placed precisely into the ribosomal P-site and the complex is ready to begin elongation.

The 2015 Colussi et al. article suggested that the eukaryotic PSIV IGR IRES can initiate translation within a prokaryotic organism (E. coli). The activity of this IRES element appears to be independent of an SD-like mechanism of initiation but is rather attributable to its manipulation of the conserved sites of the ribosomal subunits. Like that in eukaryotes, alterations of the 3-D structure of the IRES reduced its capability to initiate translation, suggesting a conserved approach of initiation, based on the structure of this initiation element, between prokaryotes and eukaryotes. 
In the current work, we used the same dual-luciferase reporter construct as that of Colussi et al. (2015) to monitor the levels of translation that each experimental construct initiated. With use of the dual-luciferase construct, the RLuc acts as an internal control, under the translational control of an SD and enhancer sequence and the FLuc is the experimental reporter, whose expression is driven by the IRES element inserted upstream. Normalized ratios of luciferase expression are calculated to remove between-sample variability caused by external influences.

\subsubsection{Capabilities of Eukaryotic IRESs to Initiate Prokaryotic Translation}

We determined whether other IRESs are capable of initiating translation in E. coli by cloning a variety of viral and cellular IRES elements, as well as several other highly structured RNA sequences, between the luciferase genes of the dual-luciferase reporter construct (Colussi et al., 2015). The yeast cellular IRESs utilized were those from FLO8, GPR1, HAP4 and NCE102 (Figure 6). FLO8, GPR1 and NCE102 have all been implicated as invasive growth genes, whereas HAP4 is involved in respiratory gene expression, with each respective IRES elements necessary for these functions (Seino et al., 2005; Gilbert et al., 2007). The viral IRESs utilized were those from the IGR of PSIV, the IGR of CrPV, CSFV and FMDV (Figure 7). The PSIV IGR IRES was used as a positive control and the CSFV IRES was used as a negative control, both of which produced results that corroborated Colussi et al. findings. CrPV is a Class 4 IRES, which folds compactly and initiates eukaryotic translation independent of any canonical initiation factors or IT AFs (Kieft, 2008). FMDV is a Class 2 IRES, which forms an extended and flexible 
conformation and initiates eukaryotic translation with the help of a significant amount of canonical initiation factors and IT AFs (Filbin and Kieft, 2009; Mailliot and Martin,2018).

(A)
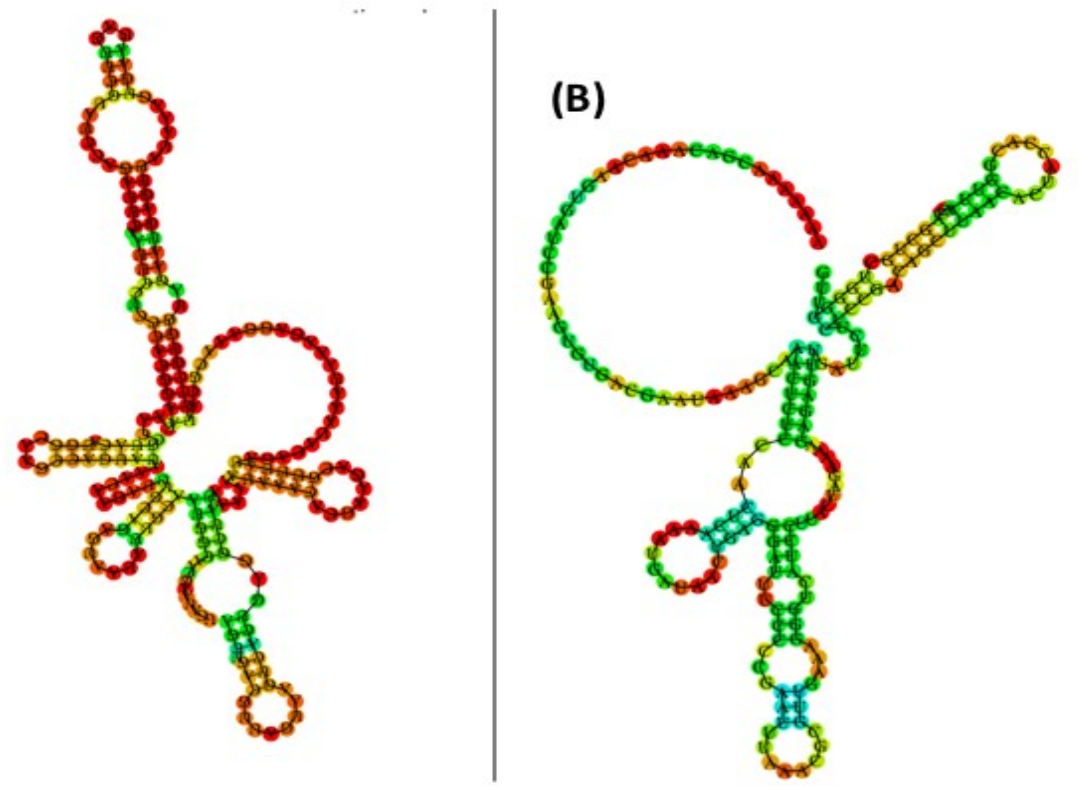

(C)

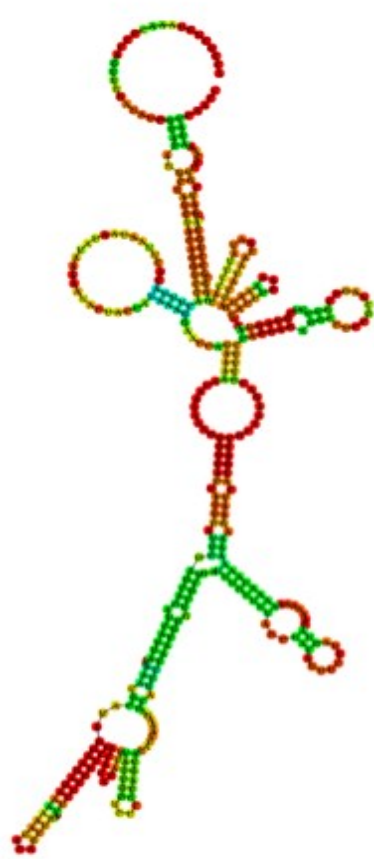

(D)

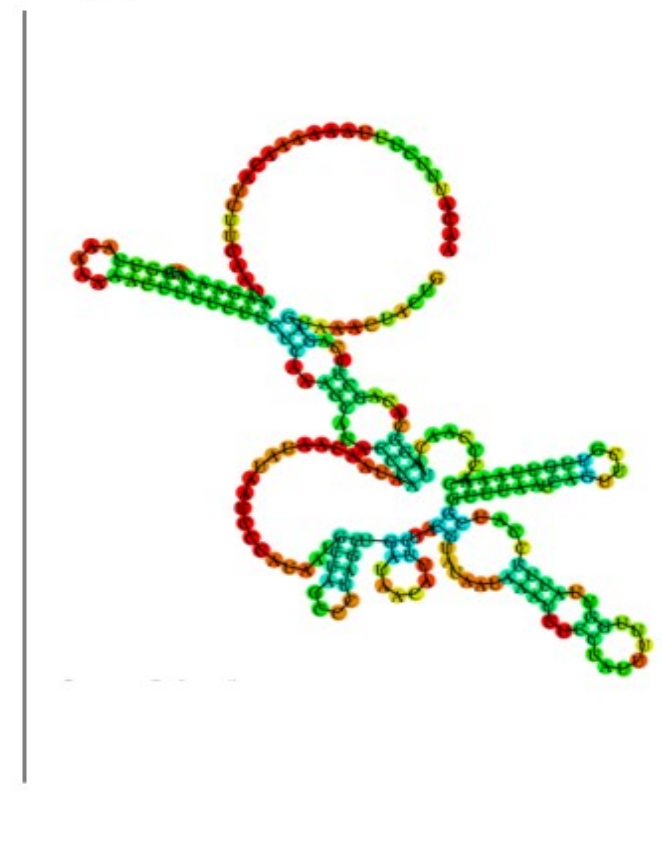

Figure 6. RNAfold predicted MFE secondary structures of the yeast cellular IRES elements used. (A) FLO8 IRES, (B) GPR1 IRES, (C) HAP4 IRES, and (D) NCE102 IRES. 
(A)

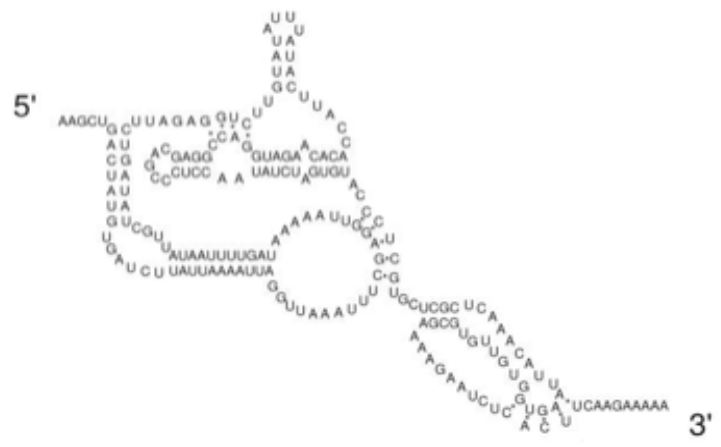

(C)

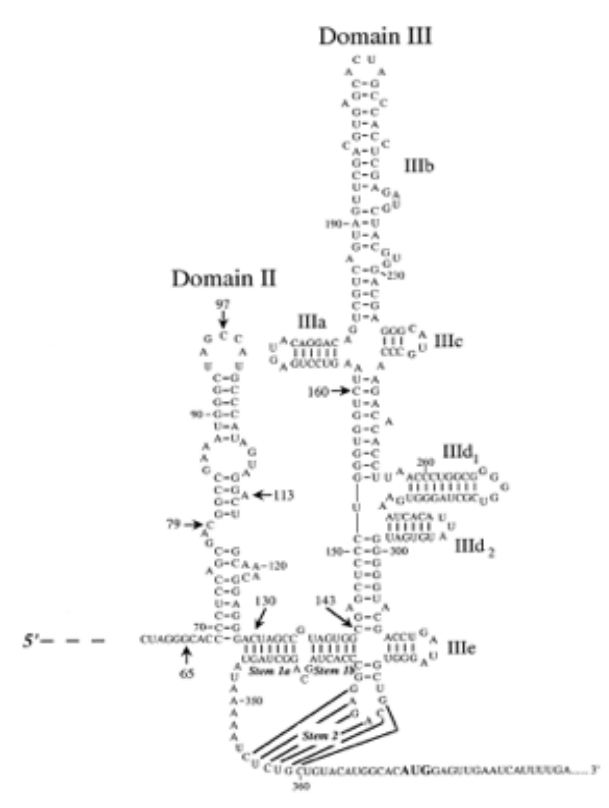

(B)

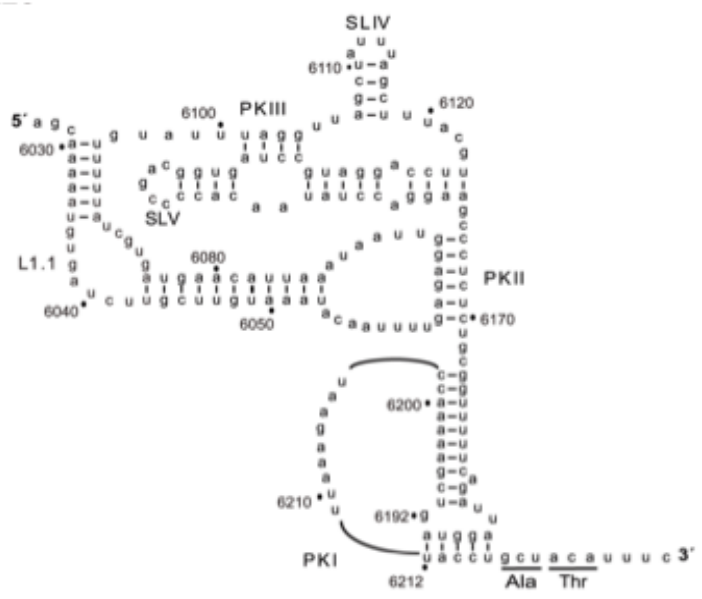

(D)

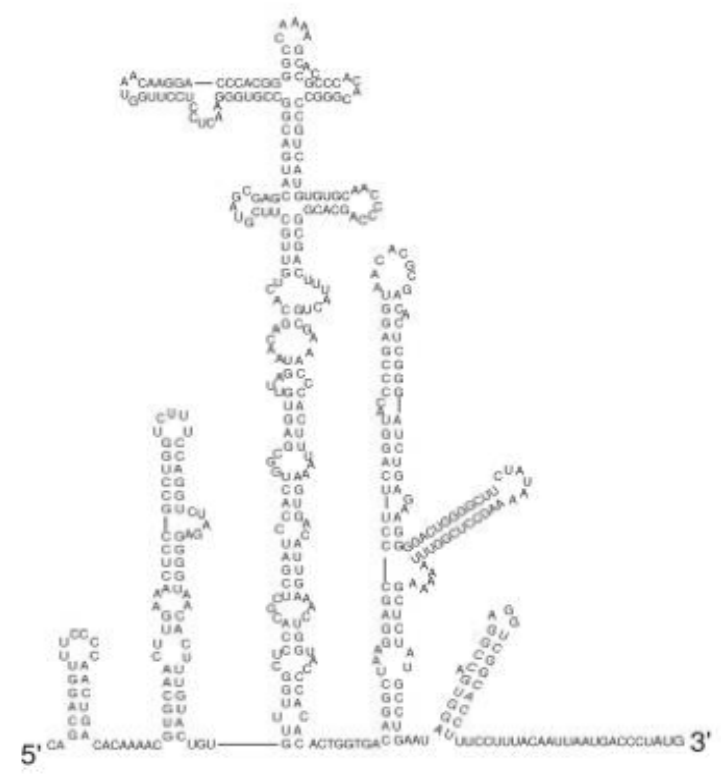

Figure 7. Secondary structures of viral IRES elements used. (A) PSIV IGR IRES, reproduced with permission from (Filbin and Kieft, 2009). (B) CrPV IGR IRES, reproduced with permission from (Wang and Jan, 2014). (C) CSFV IRES, reproduced with permission from (Fletcher and Jackson, 2002). (D) FMDV IRES, reproduced with permission from (Filbin and Kieft, 2009). 
Finally, to guarantee that not just any structured RNA element can initiate prokaryotic translation, IRES elements from $F L O 8$ and GPRI were inserted in-between the SpeI and NcoI restrictions sites of the dual-luciferase construct in reverse (Figure 8). The reversed cellular IRES elements possess an absence of IRES functionality, but maintain a stable secondary structure (Xia, 2007; Xia and Holcik, 2009). 
(A)

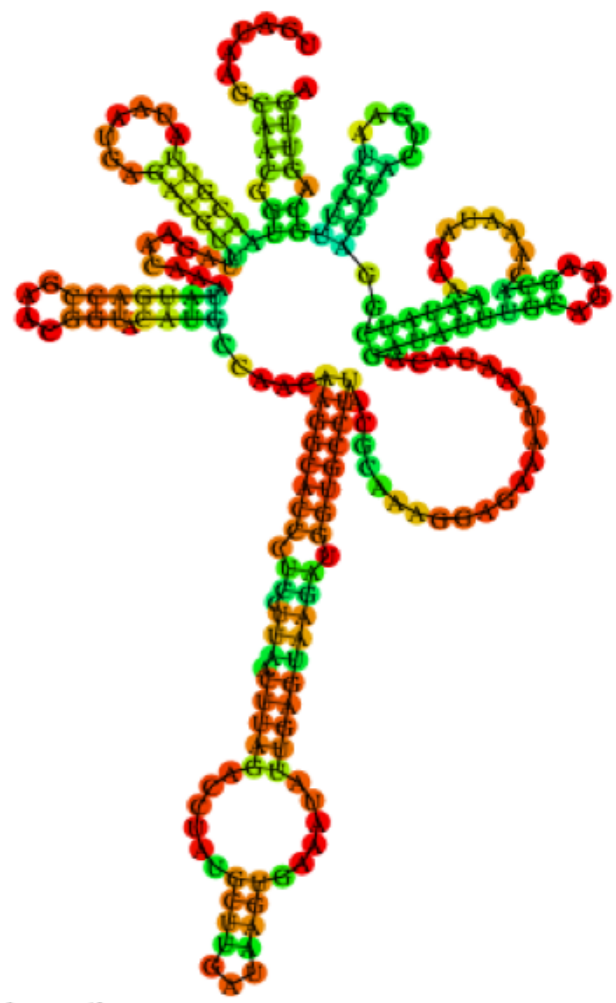

(B)

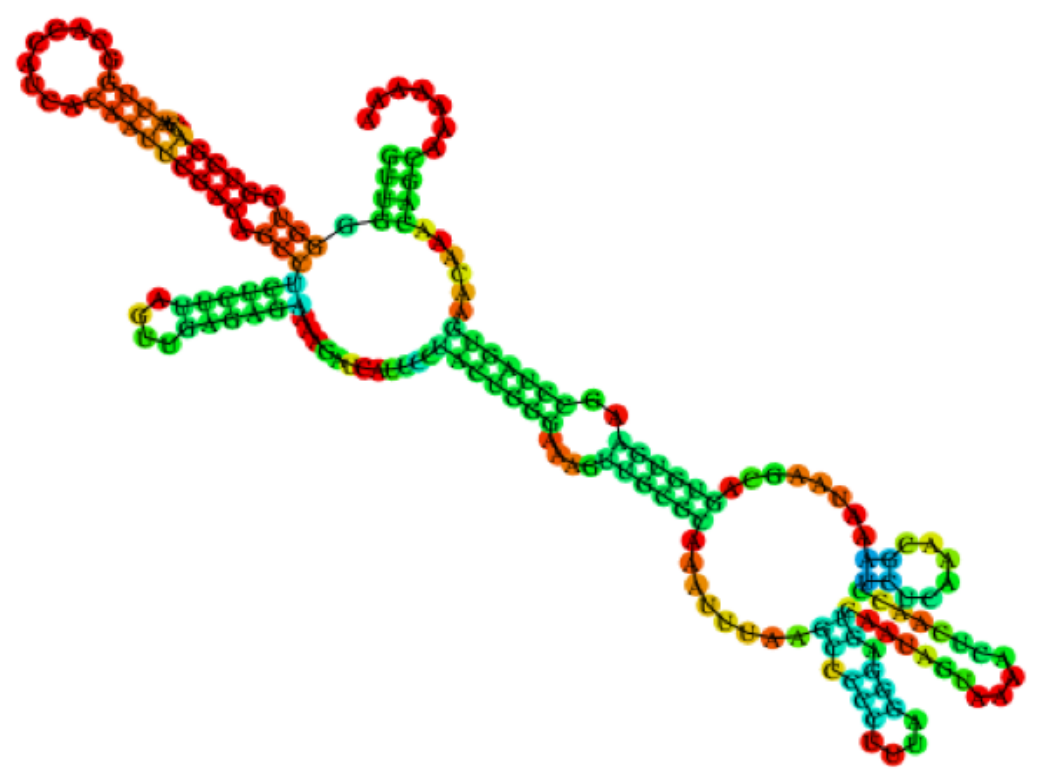

Figure 8. RNAfold predicted MFE secondary structures of reverse inserted yeast cellular IRES elements used. (A) FLO8 reverse structured RNA element, and (B) GPR1 reverse structuredRNA element. 
Based on the normalized luciferase expression data obtained, it appears that the ability of the PSIVIGR IRES to initiate prokaryotic translation is a distinct property that is not common between the other IRES elements employed (Figure 9). The cellular IRES elements, such as those from NCE102, HAP4 and FLO8, show the highest levels of IRES activity via normalized luciferase expression, at $25 \%, 23 \%$ and $12 \%$ of that produced by PSIV, respectively. While not negligible results, these values are not significant enough to provide definitive evidence that these IRES elements can drive initiation of prokaryotic translation. At the lower end, other IRES elements, such as those from GPR1, FMDV and CrPV, exhibited negligible results, at just $1 \%, 0.2 \%$ and $1 \%$ of that produced by PSIV. Taken into consideration with the inconsequential expression data generated from the insertion of highly structured RNA elements, all approximately $2 \%$ of PSIV expression, these results indicate that prokaryotic initiation is not strictly dependent on just any complex secondary structure attempting to sequester ribosomal subunits. 


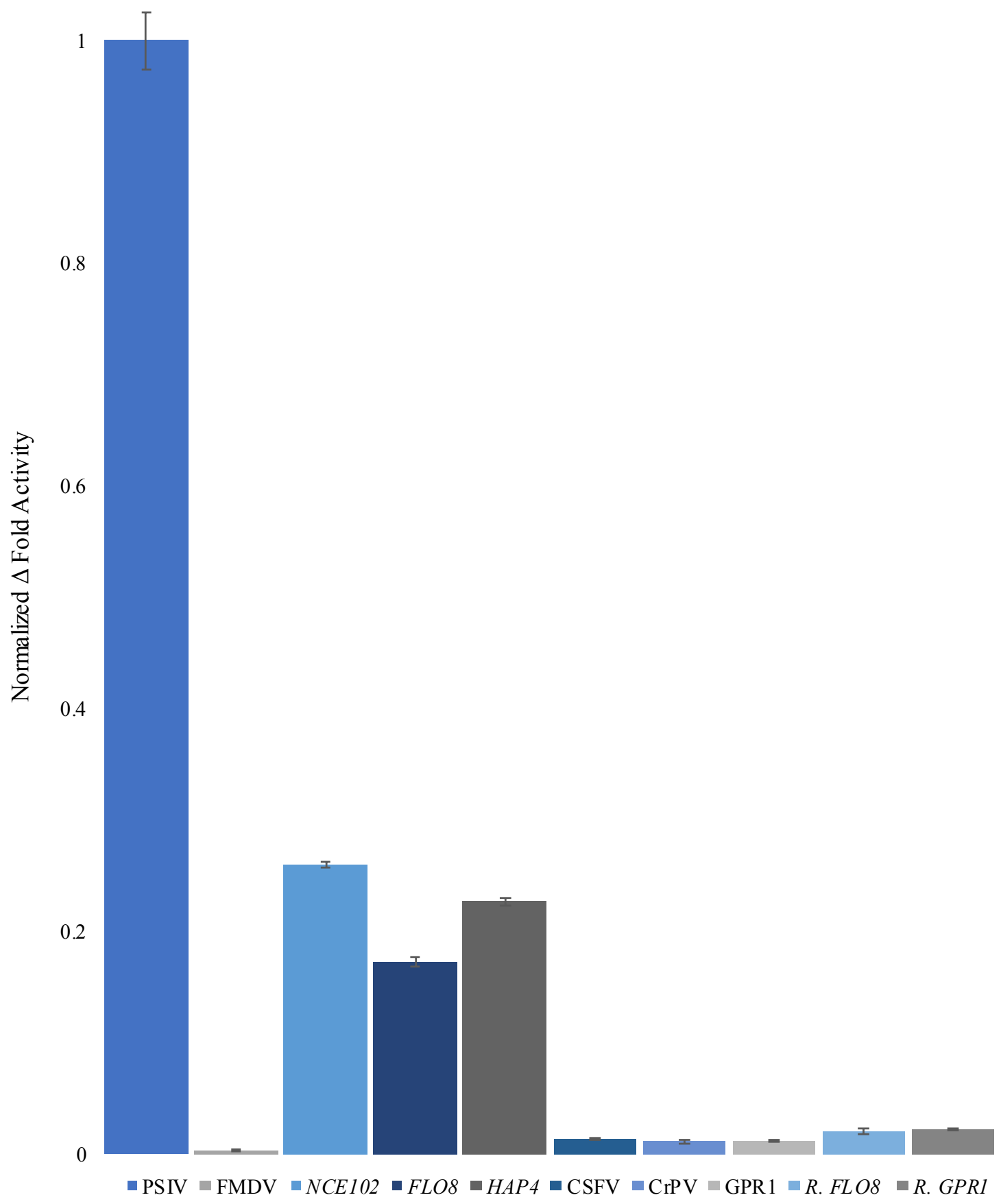

Figure 9. Normalized fold change of luciferase expression illustrating the difference in ability of various IRES and RNA elements to express FLuc relative to the SD and enhancer controlled RLuc, compared to the PSIV construct, within E. coli. Each experimental construct consists of a normalized average generated from a minimum of four biological and three technical replicates. The error bars represent the standard error of the average values. ANOVA ( $F=316.7, p<0.001)$ followed by post hoc Tukey test showed that PSIV IGR IRES activity differed significantly from each of the remaining constructs $(\mathrm{p}<0.0001)$. 


\subsubsection{Truncation of Domain III}

Colussi et al. had indicated that the structural characteristics of the PSIV IGR IRES element enabled it to initiate prokaryotic translation, and that its structural integrity is paramount to its functionality. To assess whether structural integrity has a fundamental purpose in the functionality of the PSIV IGR IRES, focus was placed on the Domain III of the IRES, following previous evidence that it is able to independently bind to the decoding groove of the 16S rRNA, as well as initiate prokaryotic translation (Zhu et al., 2011; Colussi et al., 2015).

To determine the relative importance of the PSIV Domain III structural integrity to its own functionality, its tertiary structure was disrupted. This disruption was achieved through truncation of the PSIVDomain III by the deletion of upstream nucleotides, which induced the destruction of PKI (Figure 10). Theoretically, this disruption should have rendered the Truncated Domain III non-functional relative to the Full-Length Domain III. The resulting expression data indicates otherwise, with the expression values from the Truncated Domain III significantly increased, at $76 \%$, from the $16 \%$ exhibited by theFullLength Domain III (Figure 11). Should the structural integrity have played a commanding role, deletion of any nucleotides from the Domain III structure would result in expression approaching zero percent. This leads us to believe that the secondary structure plays a reduced role in the IRES mechanism than previously thought. 
(A)

$U_{\mathrm{G}} \mathrm{C}$
(B)

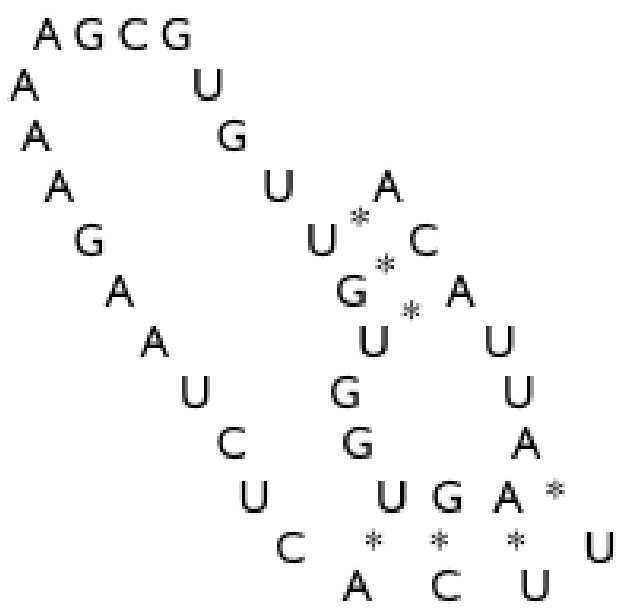

Figure 10. (A) Visual representation of the PSIV Full-Length Domain III. Sequences forming Domains I and II were deleted. (B) Visual representation of the PSIV Truncated Domain III, whereby the first 11 nucleotides were removed from the 5' end of the Full-Length Domain III sequence. 


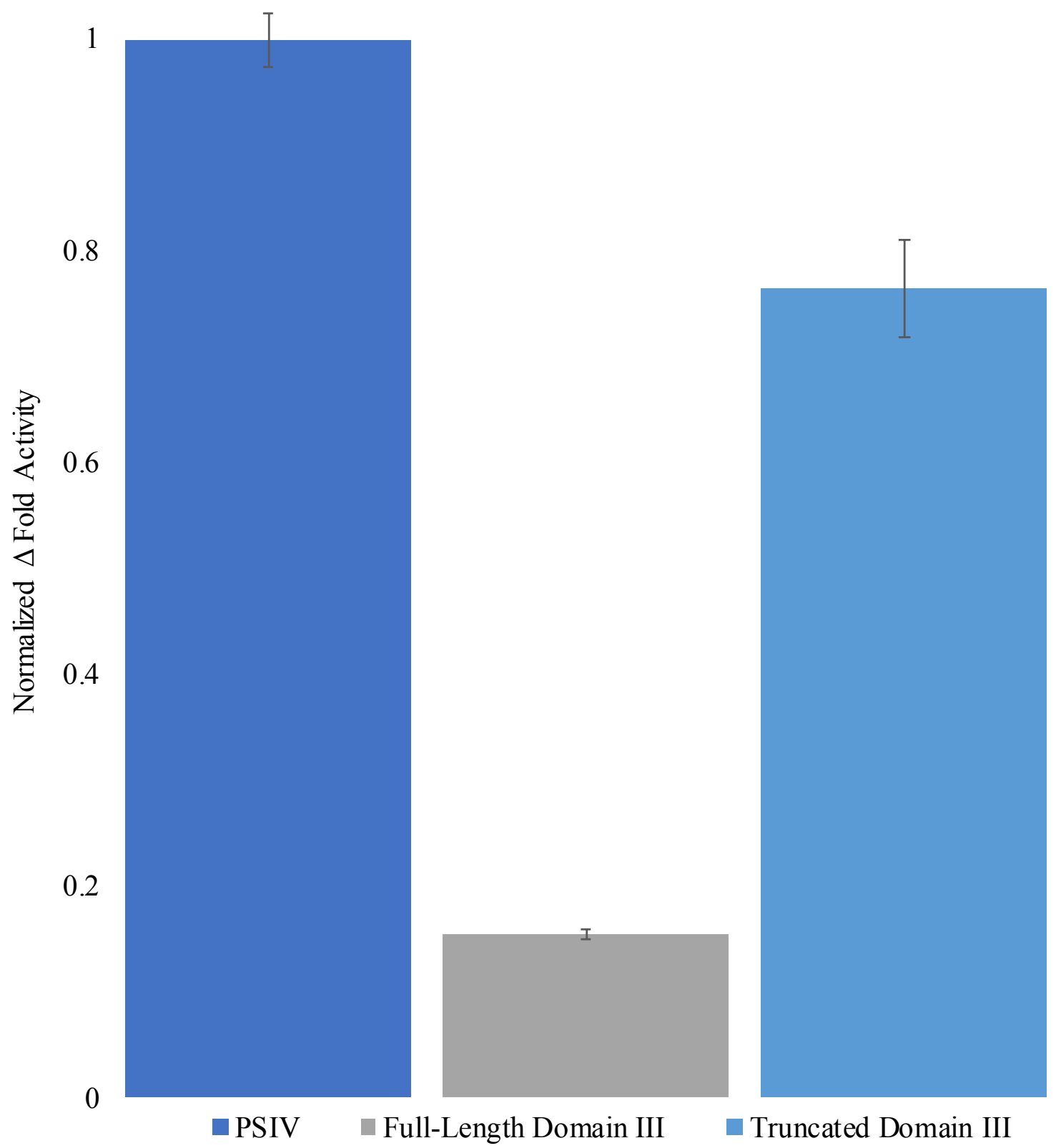

Figure 11. Normalized fold change of luciferase expression illustrating the difference in ability of the Full-Length and Truncated Domain III elements to express FLuc relative to the SD and enhancer controlled RLuc, compared to the PSIV construct, within E. coli. Each experimental construct consists of a normalized average generated from a minimum of four biological and three technical replicates. The error bars represent the standard error of the average values. ANOVA $(\mathrm{F}=55.37, \mathrm{p}<0.0001)$ followed by post hoc Tukey test showed that IRES activity of each construct differed significantly from each of other constructs $(\mathrm{p}=0.0062$ and $\mathrm{p}<0.0001(\mathrm{x} 2))$. 
Importantly, while the sequence of the Full-Length Domain III we had created matches relative to the sequence of the Full-Length Domain III published by Colussi et al., we were unable to replicate the normalized luciferase expression results put forth in the paper by Colussi et al (2015). The normalized luciferase data of the Full-Length Domain III we expressed was $16 \%$ of that of the PSIV IGR IRES, which is considerably lower than what they had reported. Although, this finding is in agreement with Colussi et al., whereby the independent Domain III is capable of initiating prokaryotic translation, yet at a significantly lower level than the entire PSIV IGR IRES.

\subsubsection{Mutating Complementary Regions}

As the other IRES elements were unable to effectively initiate prokaryotic translation and the structural integrity of Domain III appears less important than originally thought, we attempted to further elucidate the mode by which this IRES initiates prokaryotic translation. We considered whether complementarity between Domain III and the 16S rRNA plays a role in the ability of Domain III to mediate bacterial translation initiation. In eukaryotes, some IRES elements initiate translation through ribosomal recruitment via base-pair complementarity with the 18SrRNA, such as with HCV and the insulin-like growth factor 1 (IGF1R) (Martinez-Salas et al., 2018). In bacteria, translation initiation is primarily achieved through the recruitment of ribosomal subunits to themRNA via base pairing to the 16SrRNA, such as with the SD and anti-SD interaction (Shine and Dalgarno, 1974; Zhu et al., 2011). Although, non-SD base-pairing interactions have also been reported to initiate prokaryotic translation, such as with the epsilon sequence (Olins and Rangwala, 1989; Golshani et al., 1997, 2004; O’Connor, 2001). 
Two regions of interest were identified within the PSIV Domain III, designated Box 1 and Box 2, that possess complementarity to 16S rRNA regions, nucleotides 932-938 and nucleotides 1465-1471, respectively (Figure 12). These regions of complementarity present on the 16S rRNA, Anti-Box 1 and Anti-Box 2, are plausible for two reasons: 1) their location within the subunit, and 2) the function associated with their location within the subunit. The nucleotides of Anti-Box 1 are a segment of the 3 ' minor domain and portion of the penultimate stem, which has been identified to contribute to the decoding process and translational fidelity (Allen \& Noller, 1991; Firpo \& Dahlberg, 1998). The nucleotides of Anti-Box 2 are a segment the 5' major domain and part of helix 28, which constitutes the neck of the small ribosomal subunit and creates a portion of the ribosomal P-site. Helix 28 has been observed to contact the SD helix during the SD-anti-SD interaction (Gualerzi and Pon, 2015). Thus, these complimentary regions on the 16SrRNA are located within vicinity of the decoding region of the subunit, and are in spatial proximity of where Domain III (and the corresponding boxes) interact within the ribosome and allow for Watson-Crick base-pairing to occur. 


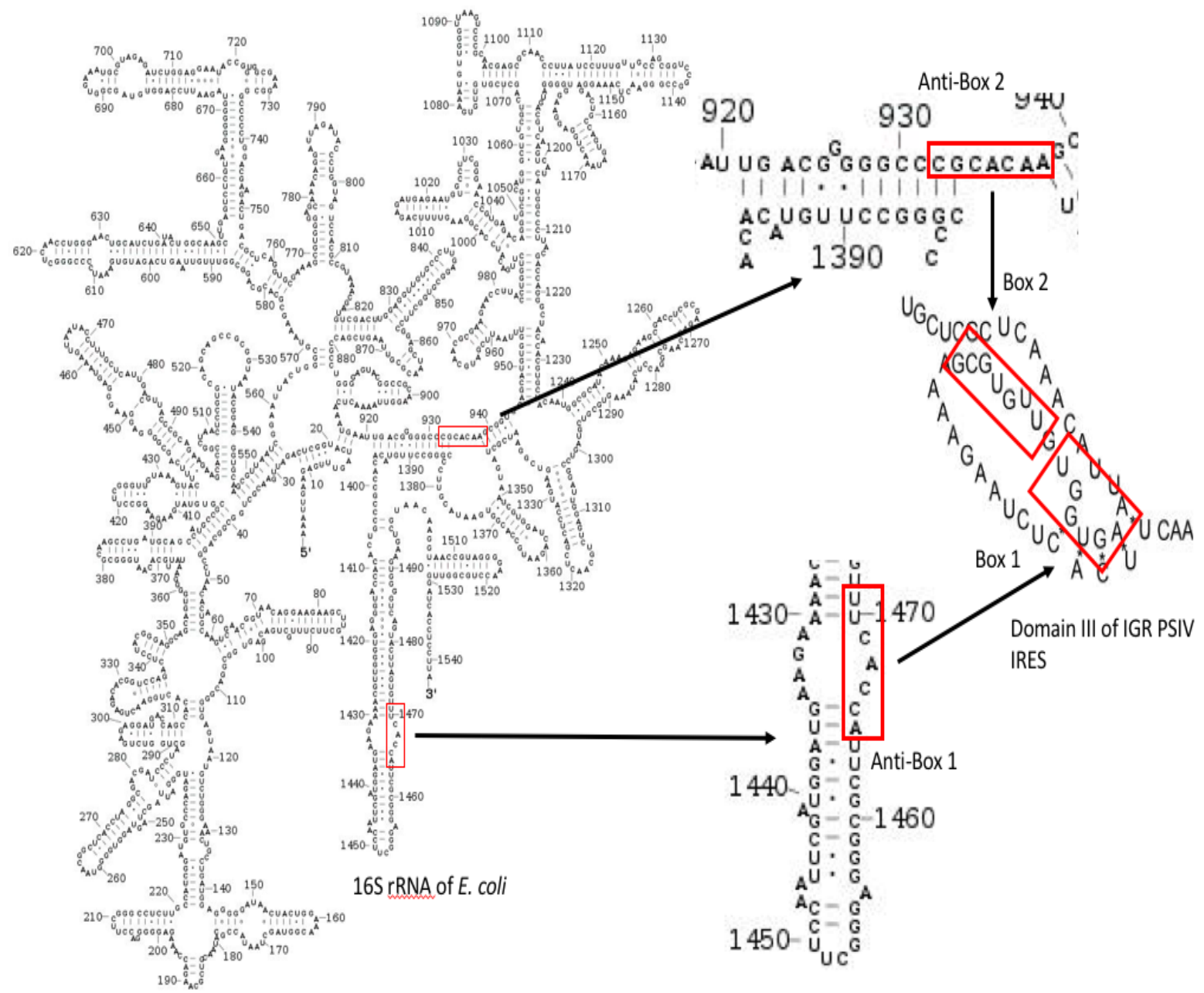

Figure 12. Complementarity illustrated between nucleotides $932-938$ and 1465-1471 of the 16S rRNA of $E$. coli and Domain III of the PSIV IGR IRES. 
Should complementarity to the $16 \mathrm{~S}$ rRNA contribute to the ability of Domain IIIto mediate bacterial translation initiation, then destroying that complementarity would abrogate the increased initiation efficiency presented by the Truncated Domain III. As anticipated, mutational analysis of the independent Truncated Domain III illustrate that the introduced mutations (designated CACT and ACTT) (Figure 13) destroying complementarity to the 16 S subunit within the Boxes reduce the ability of the mutant IRES elements to express FLuc (Figure 14). These findings suggest that the complementary regions may assume some responsibility in the ability of the PSIV IGR IRES to mediate prokaryotic translation.

(A)

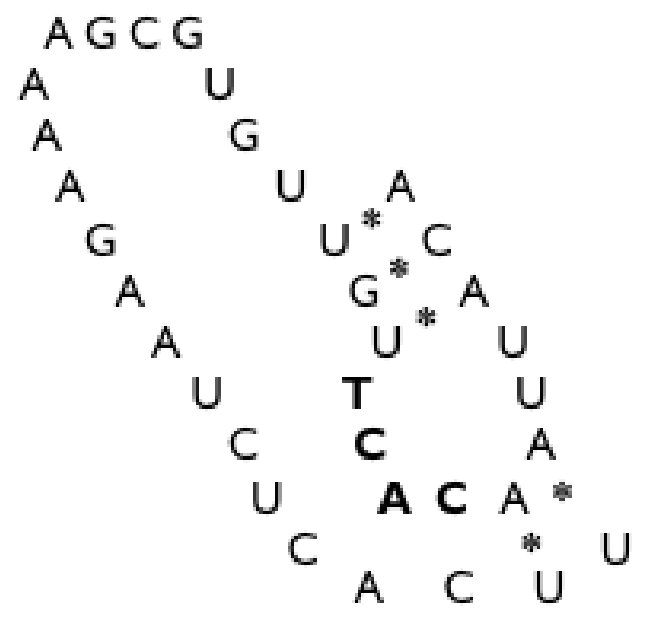

(B)

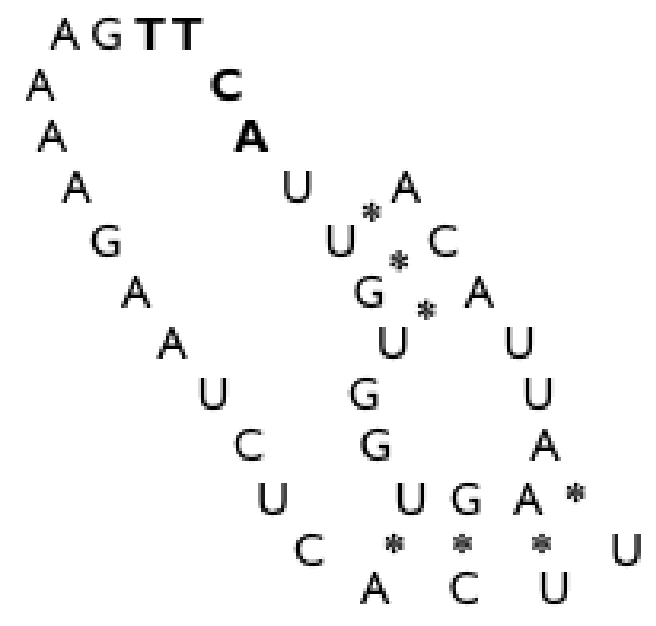

Figure 13. (A) Visual representation of the Truncated Domain III bearing the introduced mutations CACT within Box I. (B) Visual representation of the Truncated Domain III bearing the introduced mutations ACTT within Box 2. 


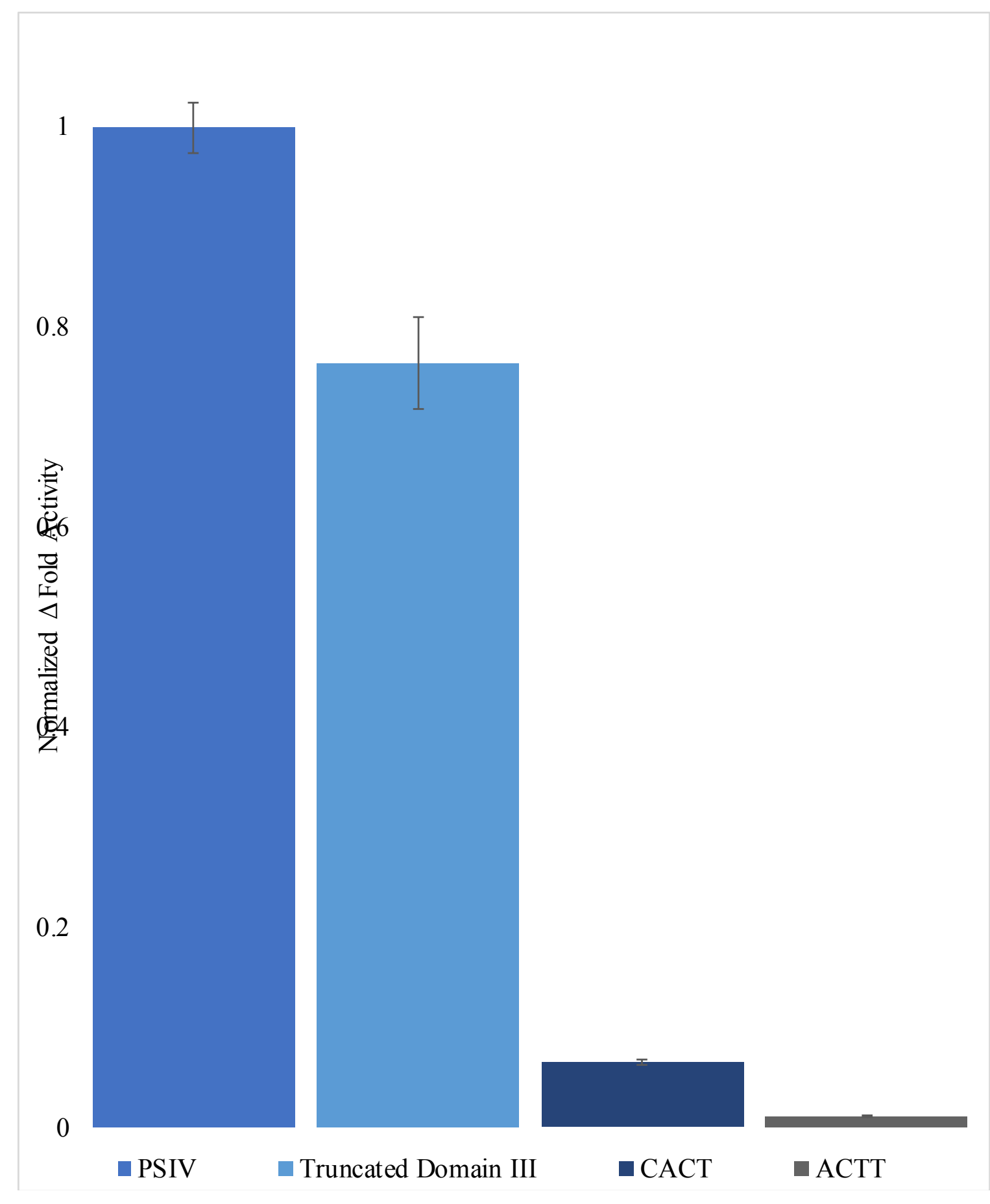

Figure 14. Normalized fold change of luciferase expression illustrating the difference in ability of mutated Truncated Domain III elements, CACT and ACTT, compared to the Truncated Domain III, to express FLuc relative to the SD and enhancer controlled RLuc, within E. coli. Each experimental construct consists of a normalized average generated from a minimum of four biological and three technical replic ates. The error bars represent the standard error of the average values. ANOVA $(\mathrm{F}=113.8, \mathrm{p}<0.0001)$ followed by post hoc Tukey test showed that IRES activity of each construct differed significantly from each of other constructs $(p=0.0026$ and $p<0.0001(x 3))$, with exception of CACT compared to ACTT, which was not-significant ( $p=0.8931)$. 


\subsection{Conclusion}

While the fundamental goals of translation initiation are the same between all cells of life, it is the most phylogenetically diverse step within the process of translation. Thisis highlighted by the minor correspondence between the mechanisms and apparatus utilized. Until recently, there has been no evidence of a molecular mechanism that can initiate translation in both prokaryotes and eukaryotes. In 2015, Colussi et al. reported that the eukaryotic PSIV IGR IRES can successfully initiate translation in the prokaryotic organism E. coli. IRES is a complex RNA secondary structure present in eukaryotic viruses and cellular mRNAs that can directly recruit ribosomes within the vicinity of the start codon. The findings of Colussi et al. indicate that this structured RNA molecule, that was believed to work exclusively in eukaryotes, can effectively circumvent domain-specific divergences. Their work states that this IRES can capitalize on conserved ribosomal features and initiate protein synthesis in both bacteria and eukaryotes. While an interesting discovery, it was not yet established if this was a unique property to the PSIV IGR IRES, or whether other IRES elements were also capable of initiating prokaryotic translation. With the results presented here, it appears that this IRES, relative to the other IRES elements employed in this study, is unique in its ability to initiate prokaryotic translation, as the other IRES elements were unable to initiate prokaryotic translation as efficiently as the PSIV IGR IRES. Our results also suggest the possibility that the structural integrity requirements for this IRES in prokaryotes differ from its functioning in eukaryotic organisms. Lastly, the break-down of complementary interactions identified by this study may imply the importance of base-pairing between Domain III and the 16SrRNA for this IRES ability to initiate prokaryotic translation. 


\section{General Discussion}

\subsection{Capabilities of Eukaryotic IRESs to Initiate Prokaryotic Translation}

Our protein expression data generated using the various eukaryotic IRES and structured RNA elements suggests that the ability of the PSIV IGR IRES to initiate prokaryotic translation is a distinct property that is not common between the other IRES elements employed in this study (Figure 9). The cellular IRES elements, such as those from $N C E 102, H A P 4$ and $F L O 8$, show the highest levels of IRES activity via normalized luciferase expression, at $25 \%, 23 \%$ and $12 \%$ of that produced by PSIV, respectively. While not negligible results, these values are not significant enough to provide definitive evidence that these IRES elements can drive initiation of prokaryotic translation. At the lower end, other IRES elements, such as those from GPRI, FMDV and CrPV, exhibited negligible results, at just $1 \%, 0.2 \%$ and $1 \%$ of that produced by PSIV. Taken into consideration with the inconsequential expression data generated from the insertion of highly structuredRNA elements, all approximately $2 \%$ of PSIV expression, indicate that prokaryotic initiation is not strictly dependent on just any complex secondary structure attempting to sequester ribosomal subunits. This is not an entirely surprising finding based on the known (and other potentially unknown) functional requirements of these elements within their eukaryotic host (Filbin \& Kieft, 2009; Kieft, 2008; Mailliot \& Martin, 2018). Consequently, this statement has limited significance. As this is fundamental and novel work, it is not currently known whether IRES elements, when forced to function within a different type of host (from eukaryotic to prokaryotic), require the same types and quantities offactorsto function optimally or are distinctly altered. For the purpose of this discussion, the presumption is that they remain the same. 
The relatively lowlevel of functionality exhibited by the yeast cellular IRESs used in this thesis, NCE102, HAP4, GPRl and FLO8 (Figure 6), were to be expected. This primarily corresponds to the lack of knowledge surrounding their functionality within their native host, such as the specifics of their secondary structure, IT AF and canonicalinitiation factor requirements, and recruitment mechanisms. This background knowledge is necessary to determine the likelihood that any of the IRESs selected could conceivably function in a prokaryotic organism. Thus, until more data regarding the requirements of these IRES elements comes to light, I am unable to provide any educated suppositions currently.

Contrary to that of the cellular IRESs, the specifics regarding the structural architecture and requirements of the non-PSIV viral IRESs used, CSFV, FMDV and CrPV (Figure 7) are reasonably well elucidated (Pilipenko et al., 2000; Pestova and Hellen, 2003; Pestova et al., 2008). The IRES element of CSFV was already determined by Colussi et al. (2015), and confirmed with this project, to be unable to initiate prokaryotic translation. As a member of the Class 3 IRESs, the CSFV IRES can directly and stably bind to the $40 \mathrm{~S}$ subunit and initiate eukaryotic translation using only a limited subset of canonical initiation factors, as well as Met-tRNAi (Pestova et al., 2001). While this IRES does not require the use of any IT AFs, it does require the addition of eIF2, GT P and initiator-tRNA to properly assemble into a $48 \mathrm{~S}$ complex at the initiation codon. This interaction is promoted by the requisite eIF3, which then further necessitated for the formation of the 80S complex on the IRES (Pestova et al., 1998; Sizova et al., 1998; Mailliot and Martin, 2018; Jaafar and Kieft, 2019). As the 40S and 30S subunits possess conserved areas of binding between the two, the problem likely does not lie in the binding 
aspect of the mechanism (Spahn et al., 2001). Where the issue might lie is the lack of requisite factors to assemble an elongation competent 70 S ribosome. While eIF2, GTP and initiator tRNA all either exist in bacteria or possess a bacterial equivalent, eIF3 or any respective counterpart is absent (Kyrpides and Woese, 1998; Roll-Mecak et al., 2000; Wilson and Cate, 2012). Thus, being an indispensable part of the mechanism, the lack of eIF3 could explain the inability of the IRES to initiate prokaryotic translation. It ispossible that the IRES can bind to the $30 \mathrm{~S}$ subunit but is unable to further assemble into a complete ribosomal complex.

Similarly, based on its functional requirements in eukaryotes, the FMDV IRES was an unlikely candidate to initiate prokaryotic translation. The FMDV IRES was determined to be an unlikely candidate partially based on its more extended structure, which makes it unable to directly bind to the $40 \mathrm{~S}$ ribosome in eukaryotes, but also based on its IT AF and canonical initiation factor requirements. The FMDVIRES requires various IT AFS, such as PTB, IT AF45 and PAG2GA, as well as several canonical initiation factors, including eIF2, eIF3, eIF4A and the C-terminus of eIF4G, to properly form the $48 \mathrm{~S}$ complex in its eukaryotic host (Andreev et al., 2007; Komar, Mazumder and Merrick, 2012; Mailliot and Martin, 2018; Martinez-Salas et al., 2018). The majority of these requirements do not possess a bacterial counterpart and thus, the lack of resourcesavailable to the IRES within E. coli make it unlikely that the IRES could function properly.

Mildly surprising of a result was the inability of the PSIV IGR IRES' conserved relative, the CrPV IGR IRES, to initiate prokaryotic translation. The Dicitroviridae IGR IRESs, like PSIV and CrPV, are highly conserved relative to one another. These elements fold into similar compact architectures and maintain the same manipulative mechanismof 
eukaryotic ribosome recruitment. These IRESs can directly and independently bind to eukaryotic ribosomal subunits and initiate translation (Mailliot and Martin, 2018; Martinez-Salas et al., 2018). Along with the fact that the phylogenetically conserved core of the ribosome includes the tRNA binding sites, the interactions of the two IRESs should be similar between the prokaryotic and eukaryotic ribosomes (Spahn et al., 2001; Zhu et al., 2011; Melnikov et al., 2012). Corresponding to this knowledge, it has been previously reported that the independent Domain III of the CrPV IGR IRES is in fact capable of binding to the 70 S ribosomal subunit, just as the PSIV IGR IRES (Zhu et al., 2011). It was identified by Zhu et al. that the PKI of Domain III in both IRESs act as a tRNA-mRNA mimic within the ribosomal P-site, with contacts established similarly in the $30 \mathrm{~S}$ subunit like those observed within the 40 S subunits. So, both PSIV and CrPV IGR IRESs possess similar architectures and both Domain IIIs can independently bind the 30 S ribosomes, but the PSIV IGR IRES can initiate prokaryotic translation and the CrPV IGR IRES cannot. This indicates that there are additional factors responsible for the functionality ofone IRES vs. the other that have not yet been addressed and cannot be easily resolved with basic commentary on the structural aspects of the IRES elements.

A potential factor playing a role in the lack of IRES functioning, other than protein requirements, could involve external or environmental influences. For example, many cellular IRESs are "activated" or predominantly utilized under a variety of (patho)physiological stress conditions, such as nutrient deprivation, hypoxia, oxidative stress and mitosis, when cap-dependent translation is limited or inhibited (Spriggs et al., 2008; Komar and Hatzoglou, 2011 a; Godet et al., 2019). Even viruses, such as FMDV and $\mathrm{PV}$, inhibit canonical translation initiation by encoding proteases that cleave eIF4G to 
increase ribosomal affinity of the viral IRES and decrease competition (Belsham, McInerney and Ross-Smith, 2000; Mailliot and Martin, 2018). As such, it is possible that the appropriate conditions surrounding the IRES elements in question have not been met as to activate their proper functioning within the prokaryotic host.

An additional distinction, albeit minor, regards the use of the AUGinitiation codon by the PSIVIGR IRES. The PSIVIGR IRES is known to initiate at a non-AUGstart codon, CAA encoding for glutamine, directly at the IRES in eukaryotes (Sasaki and Nakashima, 2000). Contrary, the PSIVIGR IRES initiates prokaryotic translation at a canonical AUG start codon that was separated from the IRES by a spacer region. Prokaryotes have been known to utilize non-AUG codons to initiate translation previously, such as GUG, UUG and AUA; with the midmost U being conserved and the 3 'Gdeemed as canonical (Gualerzi and Pon, 2015). That being said, neither triplet-codon encoding glutamine, CAA andCAG, possesses a central $\mathrm{U}$, which could explain the use of a canonical start codon. It does lead to the speculation of whether another initiation codon could be recognized and utilized to any degree, whether by this IRES or others.

\subsection{Truncation of Inde pende nt Domain III}

Colussi et al. had indicated that the structural characteristics of the PSIV IGR IRES element enabled it to initiate prokaryotic translation, and that its structural integrity is paramount to its functionality. To assess whether structural integrity has a fundamental purpose in the functionality of PSIVIGR IRES, focus was placed on the Domain III of the IRES, following previous evidence that it is able to independently bind to the decoding 
groove of the 16S rRNA, as well as initiate prokaryotic translation (Zhu et al., 2011; Colussi et al., 2015).

Based on the normalized expression data generated from the Truncated Domain III relative to the Full-Length Domain III, it appears that the 3-D structure and structural integrity of the IRES is less significant in prokaryotes than eukaryotes. The disruption of the independent Domain III involved its truncation by the deletion of few upstream nucleotides (Figure 10). This subsequently destroyed the PKI of Domain III, reducing its 3-D structure and extending its 2-D structure. Theoretically, this disruption should have rendered the Truncated Domain III non-functional, as the PKI was of highlighted importance due to its apparent ability to mimic a mRNA-tRNA interaction within the ribosomal P-site, and this interaction would consequently be spatially modified. The resulting expression data indicates otherwise, with the expression values from the Truncated Domain III considerably increased, at $76 \%$, from the $16 \%$ exhibited by the FullLength Domain III (Figure 11). Should the structural integrity have played a commanding role, deletion of any nucleotides from the Domain III structure would result in expression approaching zero percent.

Importantly, this truncation was already on top the removal of the first two domains (which house the stem loops and PKII and III, which were cited as being important), thus the structural integrity of the IRES was completely abolished and it would be assumed the luciferase data would reflect this. The expression data actually indicates otherwise based on the increased ratio relative to the Full-Length Domain III. 


\subsection{Induced Mutations of Complementary Regions}

Luciferase expression data presented of the mutated T runcated Domain III elements illustrate that mutations destroying complementarity to the $16 \mathrm{~S}$ subunit within the Boxes (designated CACT and ACT T) (Figure 12 and Figure 13) reduce the ability of the mutant IRES elements to initiate translation (Figure 14). These findings suggest that the identified complementary regions, Box 1 and Box 2, may assume some responsibility in the ability of the PSIV IGR IRES to mediate prokaryotic translation.

Box 1 and Box 2 are regions present in the PSIV Domain III that possesses complementarity to 16S rRNA regions, nucleotides 932-938 and nucleotides 1465-1471, respectively (Figure 12). These regions of complementarity present on the 16S rRNA, Anti-Box 1 and Anti-Box 2, are plausible for two reasons: 1) their location within the subunit, and 2) the function associated with their location within the subunit. The nucleotides of Anti-Box 1 are a segment of the 3' minor domain and portion of the penultimate stem, which has been identified to contribute to the decoding process and translational fidelity (Allen \& Noller, 1991; Firpo \& Dahlberg, 1998). The nucleotides of Anti-Box 2 are a segment the 5' major domain and part of helix 28, which constitutes the neck of the small ribosomal subunit and creates a portion of the ribosomal P-site. Helix 28 has been observed to contact the SD helix during the SD-aSD interaction (Gualerzi and Pon, 2015). Thus, these complimentary regions on the $16 \mathrm{~S}$ rRNA are located within vicinity of the decoding region of the subunit, and are in proximity of where Domain III (and the corresponding boxes) interact within the ribosome and can allow for Watson-Crick base-pairing to occur. 
Initiation of translation via the help of base-pairing not an uncommon mechanism of action. In eukaryotes, some IRESs initiate translation through ribosomal recruitment via base-pair complementarity with the $18 \mathrm{~S}$ rRNA, such as HCV and the cellular IRES of the insulin-like growth factor 1 (IGF1R) (Martinez-Salas et al., 2018). In bacteria, translation initiation is primarily achieved through the recruitment of ribosomal subunits to themRNA via base pairing to the $16 \mathrm{SrRNA}$, such as with the SD and anti-SD interaction (Shine and Dalgarno, 1974; Zhu et al., 2011). Although, non-SD base-pairing interactions have also been reported to initiate prokaryotic translation, such as with the epsilon sequence (Olins and Rangwala, 1989; Golshani et al., 1997, 2004; O’Connor, 2001).

Furthermore, regarding the mechanism of action, the functionality of the PSIVIGR IRES allows it to recruit and bind the ribosome directly, both in prokaryotes and eukaryotes. Following ribosomal subunit binding in E. coli, the ribosome was noted to reposition to the downstream AUG of the FLuc mRNA. This is a deviation from the mechanism of the PSIV IGR IRES observed in eukaryotes. Is it possible that the repositioning of the ribosome resembles a scanning mechanism that is present (not ofClass 4 IRESs) in eukaryotes? Or is it possible that the PSIV IGR IRES resembles and implements itself like a larger SD sequence to the ribosome? Dicistroviridae IRESs are quite small relative to other IRES elements, but are still roughly 200 nucleotides in size, which is much larger than the average $\mathrm{SD}$ consensus sequence. The spacer region present following the IRES element is also much longer than is common or optimal in E. coli. Importantly though, the identified Anti-Box 2 of the $16 \mathrm{~S}$ rRNA contacts the SD helix during the SD-anti-SD interaction, and as PKI of Domain III is observed to interact with 
the P-site of the 16S rRNA, with proposed base-pairing potentially at play, arguing that this IRES mimics an SD sequence to the ribosome is not implausible.

\subsection{Conclusions}

While the fundamental goals of translation initiation are the same between all cells of life, it is the most phylogenetically diverse step within the process of translation. Thisis highlighted by the minor correspondence between the mechanisms and apparatus utilized. Until recently, there has been no evidence of a molecular mechanism that can initiate translation in both prokaryotes and eukaryotes. In 2015, Colussi et al. reported that the PSIV IGR IRES can successfully initiate translation in the prokaryotic organism E. coli. IRES is a complex RNA secondary structure present in eukaryotic viruses and mRNAs that can directly recruit ribosomes within the vicinity of the start codon. The findings ofColussi et al. indicate that this structured RNA molecule, that was believed to work exclusively in eukaryotes, can effectively circumvent domain-specific divergences. Their work statesthat this IRES can capitalize on conserved ribosomal features and initiate protein synthesis in both bacteria and eukaryotes. While an interesting discovery, it was not yet established if this was a unique property to the PSIV IGR IRES, or whether other IRES elements were also capable of initiating prokaryotic translation. With the results presented here, it appears that this IRES, relative to the other IRES elements employed in this study, is unique in its ability to initiate prokaryotic translation, as the other IRES elements were unable to initiate prokaryotic translation as efficiently as the PSIV IGR IRES. Our results also suggest the possibility that the structural integrity requirements for this IRES in prokaryotes differfrom its functioning in eukaryotic organisms. Lastly, the break-down of complementary 
interactions identified by this study may imply the importance of base-pairing between Domain III and the 16S rRNA for this IRES' ability to initiate prokaryotic translation.

\subsection{Future Directions}

The identification of an IRES element that is functional in both prokaryotes and eukaryotes is a significant discovery. While the results of this thesis indicate that the ability of the PSIVIGR IRES to initiate prokaryotic translation is a distinct property, only a very minor subset of IRES elements were tested. Thus, in order to fully elucidate the scope of IRES functionality in a prokaryotic host, many more IRES elements (both viral and cellular) must be tested and verified. Not only that, but trials with these elements must also involve variable features, such as spacer length following the IRES element and start codon choice. Particularly, the external and environmental influences exerting effect on thehost organism must be altered as to understand the specifics surrounding differential IRES function and hypothetical activation.

Mechanistically, focus should be placed towards understanding how altering the secondary structure of this IRES can subsequently affect its ability to initiate prokaryotic translation, for the whole PSIV IGR IRES, as well as further work on the specifics surrounding Domain III. More research must also be done regarding the complementary regions present within the Domain III of the PSIVIGR IRES and the influences they may have on the potential IRES mechanism. This is particularly directed towards the speculation that the PSIV IGR IRES functions similarly to an SD sequence within E. coli.

The statement of "ability to initiate prokaryotic translation" that was included several times within this thesis is a sizeable, yet currently unsubstantiated statement. $E$. 
coli, while a model organism, is just one species of bacteria and the scope this statement refers to wider range of prokaryotic organisms, that has not yet been validated. Thus, ifthis IRES element does exploit the conserved portion of the ribosome based on its structural architecture, it should be able to do so in other bacterial species; where protein composition, protein functions, gene expression control, and other variables can be dissimilar relative to E. coli.

Further regarding the statements made surrounding this discovery, proclamations indicating that the PSIV IGR IRES' ability to function in both eukaryotes and a prokaryote is "conservation of initiation indicative of an early RNA World" is also an unsubstantiated claim that must be investigated. If there is conservation, why have we not encountered IRES elements with their corresponding mechanisms in bacterial mRNAs previously? And why do bacteriophages not use a similar mechanism to infect host cells if it is a functional mechanism, and one that is clearly popular and efficient, as evidenced by eukaryotic virus utilization? It is important to consider that forcing an element or mechanism to function in a non-native host is great in the pursuit of biotechnological advancement but yieldsnothing organic towards understanding native functionality of the organisms, mechanisms or structure in question.

The work presented within this thesis, and the work done by Colussi et al. (2015) represents just an infinitesimal fragment of the knowledge that is yet to be discovered regarding IRES function. And as with all fundamental work, significant trial and error, and exploration must be done to understand the greater picture. 


\section{References}

Allen, G. S. and Frank, J. (2007) 'Structural insights on the translation initiation complex: Ghosts of a universal initiation complex', Molecular Microbiology, 63(4), pp. 941-950. doi: 10.1111/j.1365-2958.2006.05574.x.

Allen, P. N. and Noller, H. F. (1991) 'A single base substitution in 16S ribosomal RNA suppresses streptomycin dependence and increases the frequency of translational errors', Cell. Elsevier, 66(1), pp. 141-148. doi: 10.1016/0092-8674(91)90146-P.

Andreev, D. E. et al. (2007) 'Differential factor requirement to assemble translation initiation complexes at the alternative start codons of foot-and-mouth disease virus RNA', RNA. Cold Spring Harbor Laboratory Press, 13(8), pp. 1366-1374. doi: 10.1261/rna.469707.

Belsham, G. J., McInerney, G. M. and Ross-Smith, N. (2000) 'Foot-and-Mouth Disease Virus 3C Protease Induces Cleavage of T ranslation Initiation Factors eIF4A and eIF4G within Infected Cells', Journal of Virology, 74(1), pp. 272-280. doi: 10.1128/jvi.74.1.272-280.2000.

Ben-Shem, A. et al. (2011) 'T he structure of the eukaryotic ribosome at $3.0 \AA$ resolution', Science, 334(6062), pp. 1524-1529. doi: 10.1126/science.1212642.

Benelli, D. and Londei, P. (2011) 'Translation initiation in Archaea: conserved and domain-specific features', Biochemical Society Transactions, 39(1), pp. 89-93. doi: $10.1042 /$ bst0390089.

Bergenhem, N. C. H. et al. (1992) 'Mutation creates an open reading frame within the 5' untranslated region of macaque erythrocyte carbonic anhydrase (CA) I mRNA that suppresses CA I expression and supports the scanning model for translation', Proceedings of the National Academy of Sciences of the United States of America. National Academy of Sciences, 89(18), pp. 8798-8802. doi: 10.1073/pnas.89.18.8798.

De Breyne, S. et al. (2009) 'Direct functional interaction of initiation factor eIF4Gwith type 1 internal ribosomal entry sites', Proceedings of the National Academy of Sciences of the United States of America. National Academy of Sciences, 106(23), pp. 9197-9202. doi: 10.1073/pnas.0900153106.

Coldwell, M. J. et al. (2000) 'Initiation of Apaf-1 translation by internal ribosome entry', Oncogene, 19(7), pp. 899-905. doi: 10.1038/sj.onc. 1203407.

Colussi, T . M. et al. (2015) 'Initiation of translation in bacteria by a structured eukaryotic IRES RNA', Nature, 519(7541), pp. 110-113. doi: 10.1038/nature14219.

Filbin, M. E. and Kieft, J. S. (2009) 'T oward a structural understanding of IRES RNA function', Current Opinion in Structural Biology. NIH Public Access, 19(3), pp. 267276. doi: 10.1016/j.sbi.2009.03.005.

Firpo, M. A. and Dahlberg, A. E. (1998) 'T he importance of base pairing in the penultimate stem of Escherichia coli 16SrRNA for ribosomal subunit association', Nucleic Acids Research, 26(9), pp. 2156-2160. doi: 10.1093/nar/26.9.2156.

Fletcher, S. P. and Jackson, R. J. (2002) 'Pestivirus Internal Ribosome Entry Site (IRES) Structure and Function: Elements in the 5' Untranslated Region Important for IRES 
Function', Journal of Virology, 76(10), pp. 5024-5033. doi: 10.1128/jvi.76.10.50245033.2002.

Gilbert, W. V. et al. (2007) 'Cap-independent translation is required for starvationinduced differentiation in yeast', Science, 317(5842), pp. 1224-1227. doi: 10.1126/science. 1144467.

Godet, A. C. et al. (2019) 'IRES Trans-Acting Factors, Key Actors of the Stress Response', International journal of molecular sciences. Multidisciplinary Digital Publishing Institute, 20(4), p. 924. doi: 10.3390/ijms20040924.

Golshani, A. et al. (1997) 'Does the epsilon sequence of phage T 7 function as an initiator for the translation of CAT mRNA in Escherichia coli?', Biochemical and Biophysical Research Communications, 236(2), pp. 253-256. doi: 10.1006/bbrc.1997.6842.

Golshani, A et al. (2000) 'Enhancing activity of epsilon in Escherichia coli and Agrobacterium tumefaciens cells.', Biochemical and biophysical research communications, 269(2), pp. 508-12. doi: 10.1006/bbrc.2000.2327.

Golshani, Ashkan et al. (2000) 'Epsilon as an initiator of translation of CAT mRNA in Escherichia coli', Biochemical and Biophysical Research Communications. Academic Press Inc., 273(2), pp. 528-531. doi: 10.1006/bbrc.2000.2958.

Golshani, A. et al. (2004) 'Escherichia coli mRNAs with strong Shine/Dalgarno sequences also contain $5^{\prime}$ end sequences complementary to domain \# 17 on the $16 \mathrm{~S}$ ribosomal RNA', Biochemical and Biophysical Research Communications. Academic Press, 316(4), pp. 978-983. doi: 10.1016/j.bbrc.2004.02.169.

Gray, N. K. and Wickens, M. (1998) 'CONT ROL OF T RANSLAT ION INITIATION IN ANIMALS', Annual Review of Cell and Developmental Biology, 14(1), pp. 399-458. doi: 10.1146/annurev.cellbio.14.1.399.

Gualerzi, C. O. and Pon, C. L. (2015) 'Initiation of mRNA translation in bacteria: Structural and dynamic aspects', Cellular and Molecular Life Sciences. Springer, 72(22), pp. 4341-4367. doi: 10.1007/s00018-015-2010-3.

Hertz, M. I. et al. (2013) 'Ribosomal Protein S25 Dependency Reveals a Common Mechanism for Diverse Internal Ribosome Entry Sites and Ribosome Shunting', Molecular and Cellular Biology, 33(5), pp. 1016-1026. doi: 10.1128/mcb.00879-12.

Hofacker, I. L. (2003) 'Vienna RNA secondary structure server', Nucleic Acids Research, 31(13), pp. 3429-3431. doi: 10.1093/nar/gkg599.

Inoue, H., Nojima, H. and Okayama, H. (1990) 'High efficiency transformation of Escherichia coli with plasmids', Gene. Elsevier, 96(1), pp. 23-28. doi: 10.1016/03781119(90)90336-P.

Jaafar, Z. A. and Kieft, J. S. (2019) 'Viral RNA structure-based strategies to manipulate translation', Nature Reviews Microbiology. NIH Public Access, 17(2), pp. 110-123. doi: 10.1038/s41579-018-0117-x.

Jackson, R. J. (2005) 'Alternative mechanisms of initiating translation of mammalian mRNAs', Biochemical Society Transactions, 33(6), pp. 1231-1241. doi:

10.1042/BST 20051231. 
Jackson, R. J. (2013) 'T he current status of vertebrate cellular mRNA IRESs', Cold Spring Harbor Perspectives in Biology, 5(2). doi: 10.1101/cshperspect.a011569.

Jackson, R. J., Hellen, C. U. T. and Pestova, T. V. (2010) 'T he mechanism of eukaryotic translation initiation and principles of its regulation', Nature Reviews Molecular Cell Biology. Nature Publishing Group, 11(2), pp. 113-127. doi: 10.1038/nrm2838.

Jan, E., Kinzy, T. G. and Sarnow, P. (2003) 'Divergent tRNA-like element supports initiation, elongation, and termination of protein biosynthesis', Proceedings of the National Academy of Sciences of the United States of America. National Academy of Sciences, 100(26), pp. 15410-15415. doi: 10.1073/pnas.2535183100.

Jenner, L. et al. (2012) 'Crystal structure of the 80 S yeast ribosome', Current Opinion in Structural Biology, 22(6), pp. 759-767. doi: 10.1016/j.sbi.2012.07.013.

Johnson, A. G. et al. (2017) 'Dynamics of IRES-mediated translation', Philosophical Transactions of the Royal Society B: Biological Sciences. The Royal Society, 372(1716). doi: $10.1098 /$ rstb.2016.0177.

Kaminishi, T . et al. (2007) 'A Snapshot of the 30S Ribosomal Subunit Capturing mRNA via the Shine-Dalgarno Interaction', Structure. Cell Press, 15(3), pp. 289-297. doi: 10.1016/j.str.2006.12.008.

Kapp, L. D. and Lorsch, J. R. (2004) 'The Molecular Mechanics of Eukaryotic Translation', Annual Review of Biochemistry. Annual Reviews 4139 El Camino Way, P.O. Box 10139, Palo Alto, CA 94303-0139, USA, 73(1), pp. 657-704. doi: 10.1146/annurev.biochem.73.030403.080419.

Kieft, J. S. et al. (2001) 'Mechanisms of internal ribosome entry in translation initiation', in Cold Spring Harbor Symposia on Quantitative Biology, pp. 277-283.

Kieft, J. S. (2008) 'Viral IRES RNA structures and ribosome interactions', Trends in Biochemical Sciences. NIH Public Access, 33(6), pp. 274-283. doi: 10.1016/j.tibs.2008.04.007.

Klinge, S. et al. (2011) 'Crystal structure of the eukaryotic 60S ribosomal subunit in complex with initiation factor 6', Science, 334(6058), pp.941-948. doi:

10.1126/science. 1211204 .

Komar, A. A. and Hatzoglou, M. (2011a) 'Cellular IRES-mediated translation: The war of IT AFs in pathophysiological states', Cell Cycle. Taylor \& Francis, 10(2), pp. 229-240. doi: $10.4161 /$ cc.10.2.14472.

Komar, A. A. and Hatzoglou, M. (2011b) 'Cellular IRES-mediated translation: The war of IT AFs in pathophysiological states', Cell Cycle, 10(2), pp. 229-240. doi: 10.4161/cc.10.2.14472.

Komar, A. A., Mazumder, B. and Merrick, W. C. (2012) 'A new framework for understanding IRES-mediated translation', Gene. NIH Public Access, 502(2), pp. 75-86. doi: 10.1016/j.gene.2012.04.039.

Kozak, M. (1987a) 'An analysis of 5'-noncoding sequences from 699 vertebrate messenger RNAs', Nucleic Acids Research, 15(20), pp. 8125-8148. doi: 10.1093/nar/15.20.8125. 
Kozak, M. (1987b) 'Effects of intercistronic length on the efficiency of reinitiation by eucaryotic ribosomes.', Molecular and Cellular Biology. American Society for Microbiology Journals, 7(10), pp. 3438-3445. doi: 10.1128/mcb.7.10.3438.

Kozak, M. (1991a) 'A short leader sequence impairs the fidelity of initiation by eukaryotic ribosomes.', Gene expression, 1(2), pp. 111-115. doi: 10.1083/jcb.115.4.887.

Kozak, M. (1991b) 'A short leader sequence impairs the fidelity of initiation by eukaryotic ribosomes.', Gene expression. Cognizant Communication Corporation, 1(2), pp. 111-115. Available at: http://www.ncbi.nlm.nih.gov/pubmed/1820208 (Accessed: 21 July 2019).

Kozak, M. (2002) 'Pushing the limits of the scanning mechanism for initiation of translation', Gene, pp. 1-34. doi: 10.1016/S0378-1119(02)01056-9.

Kwak, K. et al. (2014) 'Impact of inhibitors and L2 antibodies upon the infectivity of diverse alpha and beta human papillomavirus types', PLoS ONE. Edited by Z.-M. Zheng, 9(5), p. e97232. doi: 10.1371/journal.pone.0097232.

Kyrpides, N. C. and Woese, C. R. (1998) 'Universally conserved translation initiation factors', Proceedings of the National Academy of Sciences of the United States of America. National Academy of Sciences, 95(1), pp. 224-228. doi: 10.1073/pnas.95.1.224.

de la Cruz, J., Karbstein, K. and Woolford, J. L. (2015) 'Functions of Ribosomal Proteins in Assembly of Eukaryotic Ribosomes In Vivo', Annual Review of Biochemistry. Annual Reviews, 84(1), pp. 93-129. doi: 10.1146/annurev-biochem-060614-033917.

Lafontaine, D. L. J. and T ollervey, D. (2001) 'The function and synthesis of ribosomes', Nature Reviews Molecular Cell Biology, pp. 514-520. doi: 10.1038/35080045.

Lang, K. J. D., Kappel, A. and Goodall, G. J. (2002) 'Hypoxia-inducible factor-1 $\alpha$ mRNA contains an internal ribosome entry site that allows efficient translation during normoxia and hypoxia', Molecular Biology of the Cell, 13(5), pp. 1792-1801. doi: 10.1091/mbc.02-02-0017.

Laursen, B. S. et al. (2005) 'Initiation of Protein Synthesis in Bacteria', Microbiology and Molecular Biology Reviews, 69(1), pp. 101-123. doi: 10.1128/mmbr.69.1.101123.2005 .

López-Lastra, M. et al. (2010) 'T ranslation initiation of viral mRNAs', Reviews in Medical Virology, 20(3), pp. 177-195. doi: 10.1002/rmv.649.

López-Lastra, M., Rivas, A. and Barría, M. I. (2005) 'Protein synthesis in eukaryotes: The growing biological relevance of cap-independent translation initiation', Biological Research, pp. 121-146. doi: 10.4067/S0716-97602005000200003.

Lozano, G. and Martínez-Salas, E. (2015) 'Structural insights into viral IRES-dependent translation mechanisms', Current Opinion in Virology, 12, pp. 113-120. doi: 10.1016/j.coviro.2015.04.008.

Mailliot, J. and Martin, F. (2018) 'Viral internal ribosomal entry sites: four classes for one goal', Wiley Interdisciplinary Reviews: RNA, 9(2). doi: 10.1002/wrna.1458.

Martinez-Salas, E. et al. (2018) 'Insights into structural and mechanistic features of viral 
IRES elements', Frontiers in Microbiology. Frontiers, 8(JAN), p. 2629. doi: 10.3389/fmicb.2017.02629.

Melnikov, S. et al. (2012) 'One core, two shells: Bacterial and eukaryotic ribosomes', Nature Structural and Molecular Biology, 19(6), pp. 560-567. doi: 10.1038/nsmb.2313.

Mével-Ninio, M. et al. (1996) 'The three dominant female-sterile mutations of the Drosophila ovo gene are point mutations that create new translation-initiator AUG codons.', Development (Cambridge, England), 122(12), pp. 4131-8. Available at: http://www.ncbi.nlm.nih.gov/pubmed/9012532 (Accessed: 27 July 2019).

Milón, P. and Rodnina, M. V. (2012) 'Kinetic control of translation initiation in bacteria', Critical Reviews in Biochemistry and Molecular Biology, pp. 334-348. doi: 10.3109/10409238.2012.678284.

Moteshareie, H. et al. (2018) 'Heavy metal sensitivities of gene deletion strains for IT T1 and RPS1 A connect their activities to the expression of URE2, a key gene involved in metal detoxification in yeast', PLoS ONE, 13(9), pp. 1-18. doi: 10.1371/journal.pone.0198704.

O'Connor, M. (2001) 'Enhancement of translation by the epsilon element is independent of the sequence of the 460 region of 16SrRNA', Nucleic Acids Research. Narnia, 29(7), pp. 1420-1425. doi: 10.1093/nar/29.7.1420.

Olins, P. O. and Rangwala, S. H. (1989) 'A novel sequence element derived from bacteriophage $\mathrm{T} 7 \mathrm{mRNA}$ acts as an enhancer of translation of the lacZ gene in Escherichia coli', Journal of Biological Chemistry, 264(29), pp. 16973-16976. Available at: http://www.jbc.org/content/264/29/16973.full.pdf(Accessed: 27 July 2019).

Pelletier, J. and Sonenberg, N. (1988) 'Internal initiation of translation of eukaryotic mRNA directed by a sequence derived from poliovirus RNA', Nature, 334(6180), pp. 320-325. doi: 10.1038/334320a0.

Pestova, T. Vet al. (1998) 'A prokaryotic-like mode of cytoplasmic eukaryotic ribosome binding to the initiation codon during internal translation initation of hepatitis $\mathrm{C}$ and classical swine fever virus RNAs', Genes and Development. Cold Spring Harbor Laboratory Press, 12(1), pp. 67-83. doi: 10.1101/gad.12.1.67.

Pestova, T. Vet al. (2001) 'Molecular mechanisms of translation initiation in eukaryotes', Proceedings of the National Academy of Sciences of the United States of America. National Academy of Sciences, 98(13), pp.7029-7036. doi: 10.1073/pnas. 111145798.

Pestova, T. Vet al. (2008) 'eIF2-dependent and eIF2-independent modes of initiation on the CSFV IRES: A common role of domain II', EMBO Journal. European Molecular Biology Organization, 27(7), pp. 1060-1072. doi: 10.1038/emboj.2008.49.

Pestova, T. V and Hellen, C. U. T . (2003) 'Translation elongation after assembly of ribosomes on the Cricket paralysis virus internal ribosomal entry site without initiation factors or initiator tRNA', Genes and Development. Cold Spring Harbor Laboratory Press, 17(2), pp. 181-186. doi: 10.1101/gad.1040803.

Pichon, X. et al. (2012) 'RNA Binding Protein/RNA Element Interactions and the Control of T ranslation', Current Protein \& Peptide Science. Bentham Science Publishers, 
13(4), pp. 294-304. doi: 10.2174/138920312801619475.

Pilipenko, E. V et al. (2000) 'A cell cycle-dependent protein serves as a template-specific translation initiation factor.', Genes \& development. Cold Spring Harbor Laboratory Press, 14(16), pp. 2028-45. Available at: http://www.ncbi.nlm.nih.gov/pubmed/10950867 (Accessed: 9 August 2019).

Pooggin, M. M., Hohn, T . and Fütterer, J. (2000) 'Role of a short open reading frame in ribosome shunt on the cauliflower mosaic virus RNA leader', Journal of Biological Chemistry. American Society for Biochemistry and Molecular Biology, 275(23), pp. 17288-17296. doi: 10.1074/jbc.M001143200.

Pooggin, M. M. and Ryabova, L. A. (2018) 'Ribosome shunting, polycistronic translation, and evasion of antiviral defenses in plant pararetroviruses and beyond', Frontiers in Microbiology. Frontiers, 9(APR), p. 644. doi: 10.3389/fmicb.2018.00644.

Ramakrishnan, V. (2002) 'Ribosome Structure and the Mechanism of Translation', Cell. Cell Press, 108(4), pp. 557-572. doi: 10.1016/S0092-8674(02)00619-0.

Ramesh, M. and Woolford, J. L. (2016) 'Eukaryote-specific rRNA expansion segments function in ribosome biogenesis', $R N A, 22(8)$, pp. 1153-1162. doi:

10.1261/rna.056705.116.

Roll-Mecak, A. et al. (2000) 'X-ray structures of the universal translation initiation factor IF2/eIF5B: Conformational changes on GDP and GT P binding', Cell. Cell Press, 103(5), pp. 781-792. doi: 10.1016/S0092-8674(00)00181-1.

Ryabova, L. A., Pooggin, M. M. and Hohn, T . (2006) 'Translation reinitiation and leaky scanning in plant viruses', Virus Research. Elsevier, 119(1), pp. 52-62. doi: 10.1016/j.virusres.2005.10.017.

Sasaki, J. and Nakashima, N. (2000) 'Methionine-independent initiation of translation in the capsid protein of an insect RNA virus', Proceedings of the National Academy of Sciences of the United States of America, 97(4), pp. 1512-1515. doi: 10.1073/pnas.010426997.

Seino, A. et al. (2005) 'T ranslational control by internal ribosome entry site in Saccharomyces cerevisiae', Biochimica et Biophysica Acta - Gene Structure and Expression. Elsevier, 1681(2-3), pp. 166-174. doi: 10.1016/j.bbaexp.2004.11.009.

Sherrill, K. W. et al. (2004) 'BCL-2 translation is mediated via internal ribosome entry during cell stress', Journal of Biological Chemistry, 279(28), pp. 29066-29074. doi: $10.1074 / \mathrm{jbc} . \mathrm{M} 402727200$.

Shi, Y. J. et al. (2005) 'Cyclin D1 and c-myc internal ribosome entry site (IRES)dependent translation is regulated by AKT activity and enhanced by rapamycin through a p38 MAPK- and ERK-dependent pathway', Journal of Biological Chemistry, 280(12), pp. 10964-10973. doi: 10.1074/jbc.M407874200.

Shine, J. and Dalgarno, L. (1974) 'The 3'-Terminal Sequence of Escherichia coli 16S Ribosomal RNA: Complementarity to Nonsense Triplets and Ribosome Binding Sites', 71(4), pp. 1-5. Available at: https://www.pnas.org/content/pnas/71/4/1342.full.pdf (Accessed: 27 July 2019).

Shultzaberger, R. K. et al. (2001) 'Anatomy of Escherichia coli ribosome binding sites', 
Journal of Molecular Biology, 313(1), pp. 215-228. doi: 10.1006/jmbi.2001.5040. Simonetti, A. et al. (2009) 'A structural view of translation initiation in bacteria', Cellular and Molecular Life Sciences, 66(3), pp. 423-436. doi: 10.1007/s00018-0088416-4.

Sizova, D. V et al. (1998) 'Specific interaction of eukaryotic translation initiation factor 3 with the 5 ' nontranslated regions of hepatitis $\mathrm{C}$ virus and classical swine fever virus RNAs.', Journal of virology. American Society for Microbiology (ASM), 72(6), pp. 4775-82. Available at: http://www.ncbi.nlm.nih.gov/pubmed/9573242 (Accessed: 5 August 2019).

Somogyi, P. et al. (1993) 'Ribosomal pausing during translation of an RNA pseudoknot.', Molecular and Cellular Biology. American Society for Microbiology Journals, 13(11), pp. 6931-6940. doi: 10.1128/mcb.13.11.6931.

Sonenberg, N. and Hinnebusch, A. G. (2009) 'Regulation of T ranslation Initiation in Eukaryotes: Mechanisms and Biological Targets', Cell. Cell Press, 136(4), pp. 731-745. doi: $10.1016 /$ j.cell.2009.01.042.

Spahn, C. M. T . et al. (2001) 'Structure of the 80S ribosome from Saccharomyces cerevisiae - tRNA-ribosome and subunit-subunit interactions', Cell. Elsevier, 107(3), pp. 373-386. doi: 10.1016/S0092-8674(01)00539-6.

Spriggs, K. A. et al. (2008) 'Re-programming of translation following cell stress allows IRES-mediated translation to predominate', Biology of the Cell. John Wiley \& Sons, Ltd (10.1111), 100(1), pp. 27-38. doi: 10.1042/bc20070098.

Stacey, S. N. et al. (2000) 'Leaky Scanning Is the Predominant Mechanism for Translation of Human Papillomavirus Type 16E7 Oncoprotein from E6/E7 Bicistronic mRNA', Journal of Virology. American Society for Microbiology (ASM), 74(16), pp. 7284-7297. doi: 10.1128/jvi.74.16.7284-7297.2000.

Steitz, J. A. and Jakest, K. (1975) How ribosomes select initiator regions in mRNA: Base pair formation between the 3' terminus of $16 S r R N A$ and the mRNA during initiation of protein synthesis in Escherichia coli (specificity in polypeptide chain initiation/RNA secondary structure), Biochemistry. Available at: https://www.pnas.org/content/pnas/72/12/4734.full.pdf (Accessed: 27 July 2019).

Stoneley, M. et al. (1998) 'C-Myc 5' untranslated region contains an internal ribosome entry segment', Oncogene, 16(3), pp. 423-428. doi: 10.1038/sj.onc.1201763.

Vimberg, V. et al. (2007) 'T ranslation initiation region sequence preferences in Escherichia coli', BMC Molecular Biology. BioMed Central, 8(1), p. 100. doi: 10.1186/1471-2199-8-100.

Wang, Q. S. and Jan, E. (2014) 'Switch from cap- to factorless IRES-dependent 0 and +1 frame translation during cellular stress and dicistrovirus infection', PLOS ONE. Edited by T. Preiss. Public Library of Science, 9(8), p. e103601. doi:

10.1371/journal.pone.0103601.

Wilson, D. N. and Cate, J. H. D. (2012) 'The structure and function of the eukaryotic ribosome', Cold Spring Harbor Perspectives in Biology. Cold Spring Harbor Laboratory Press, 4(5), p. 5. doi: 10.1101/cshperspect.a011536. 
Wool, I. G. (1979) 'T he Structure and Function of Eukaryotic Ribosomes', Annual Review of Biochemistry. Annual Reviews 4139 El Camino Way, P.O. Box 10139, Palo Alto, CA 94303-0139, USA, 48(1), pp. 719-754. doi:

10.1146/annurev.bi.48.070179.003443.

Xia, X. (2007) 'Internal ribosomal entry site lacks secondary structure', Nature Precedings. doi: 10.1038/npre.2007.1248.1.

Xia, X. and Holcik, M. (2009) 'Strong eukaryotic IRESs have weak secondary structure', PLoS ONE. Edited by T. Preiss. Public Library of Science, 4(1), p. e4136. doi: 10.1371/journal.pone.0004136.

Yusupova, G. Z. et al. (2001) 'The path of messenger RNA through the ribosome', Cell. Elsevier, 106(2), pp. 233-241. doi: 10.1016/S0092-8674(01)00435-4.

Zhu, J. et al. (2011) 'Crystal structures of complexes containing domains from two viral internal ribosome entry site (IRES) RNAs bound to the 70S ribosome', Proceedings of the National Academy of Sciences of the United States of America, 108(5), pp. 18391844. doi: 10.1073/pnas.1018582108.

Zinoviev, A., Hellen, C. U. T . and Pestova, T. V (2015) 'Multiple Mechanisms of Reinitiation on Bicistronic Calicivirus mRNAs', Molecular Cell. NIH Public Access, 57(6), pp. 1059-1073. doi: 10.1016/j.molcel.2015.01.039.

Zuker, M. and Stiegler, P. (1981) 'Optimal computer folding of large RNA sequences using thermodynamics and auxiliary information', Nucleic Acids Research, 9(1), pp. 133-148. doi: 10.1093/nar/9.1.133. 


\section{Appendix 1}

Table 2. Oligonucleotide primers used in the PCR amplification of viral and cellular IRESs and structured RNA elements for their insertion into the dual-luciferase pET30a reporter vector. The first four nucleotides in the forw ard and reverse primers are leader sequences. ACTAGT is the SpeI restriction site and CCATGG is the NcoI restriction site.

\begin{tabular}{|c|c|}
\hline Construct & Primer Seque nce \\
\hline \multirow{2}{*}{ FMDV } & F: GATC ACTAGT AGCAGGTTTCCCCAATGACAC \\
\hline & R: GATC CCATGG TTGCAAAGGAAAGGTGCCGA \\
\hline \multirow{2}{*}{$\mathrm{CrPV}$} & F: GATC ACTAGT AAAGCA AAAATGTGAT CTTGCTTGTA AATACAATTTTGA \\
\hline & R: GATC CCATGG GAAATGTAGCAGGTAAATTTCTTAGG \\
\hline \multirow{2}{*}{ FLO8 } & F: GATC ACTAGT AGTTGACGTTAGTAAGTCACTGAGG \\
\hline & R: GATC CCATGG CACTATTCGTTGCCATT \\
\hline \multirow{2}{*}{ GPRl } & F: GATC ACTAGT AAAAAAACGACAAACAAGTGATCCGAAG \\
\hline & R: TAAT CCATGG CAACCCCAGCAGCTGGTTAA \\
\hline \multirow{2}{*}{ HAP4 } & F: GGTC ACTAGT ACCTCTCTAAACCCCAGTTTTATATC \\
\hline & R: GATC CCATGG GGTTGGTATTTGGGG \\
\hline \multirow{2}{*}{ NCE102 } & F: GGTC ACTAGT AACATTTCTTTAAAAAACATCTTCAATAAGAAAAATCGG \\
\hline & R: TAAT CCATGG CAGTAGTTTACTCTGGAGCTGTGC \\
\hline \multirow{2}{*}{$\begin{array}{c}F L O 8 \\
\text { reverse }\end{array}$} & F: TA CCATGG AGTTGACGTTAGTAAGTCA \\
\hline & R: TA ACTAGT CACTATTCGTTGCCATTGC \\
\hline \multirow{2}{*}{$\begin{array}{c}G P R 1 \\
\text { reverse }\end{array}$} & F: TA CCATGG AAAAAAACGACAAACAAGTGA \\
\hline & R: TA ACTAGT CAACCCCAGCAGCTGG \\
\hline
\end{tabular}

